\title{
Conformal Symmetry and the Three Point Function for the Gravitational Axial Anomaly
}

\author{
Jiannis Pachos* and Ricardo Schiappa ${ }^{\dagger}$ \\ Center for Theoretical Physics and Department of Physics \\ Massachusetts Institute of Technology, 77 Massachusetts Ave. \\ Cambridge, MA 02139, U.S.A.
}

(MIT-CTP $\sharp 2760$, hep-th/9807128, June 1998)

\begin{abstract}
This work presents a first study of a radiative calculation for the gravitational axial anomaly in the massless Abelian Higgs model. The two loop contribution to the anomalous correlation function of one axial current and two energy-momentum tensors, $\left\langle A_{\alpha}(z) T_{\mu \nu}(y) T_{\rho \sigma}(x)\right\rangle$, is computed at an order that involves only internal matter fields. Conformal properties of massless field theories are used in order to perform the Feynman diagram calculations in the coordinate space representation. The two loop contribution is found not to vanish, due to the presence of two independent tensor structures in the anomalous correlator.
\end{abstract}

PACS: 04.62.+v, 11.10.-z, 11.15.Bt, 11.25.Db, 11.25.Hf, 11.30.Rd, 11.40.Ha

Keywords: Anomalies, Perturbation Theory, Conformal Symmetry, Quantum Gravity

*E-mail address: pachos@ctp.mit.edu

$\dagger$ E-mail address: ricardos@ctp.mit.edu 


\section{INTRODUCTION AND DISCUSSION}

Perturbation theory anomalies have been known for a long time, starting with the work by Bell and Jackiw [1] and by Adler [2] concerning anomalies in gauge theories. The fact that there are no radiative corrections to the one loop result for the anomaly has been countlessly proven or brought into question since the early work of Adler and Bardeen [3]. For this reason, explicit calculations of possible radiative corrections to the one loop anomaly are of particular interest.

Using a method proposed by Baker and Johnson [4], Erlich and Freedman recently performed such an explicit calculation for the two loop contribution of the anomalous correlation function $\left\langle A_{\mu}(x) A_{\nu}(y) A_{\rho}(z)\right\rangle$ of three chiral currents, in the Abelian Higgs model and in the Standard Model [5]. In here, we wish to extend such calculation to the case of a gravitational background.

The Adler-Bell-Jackiw anomaly concerns the divergence of the axial current in a gauge field background. The calculation of the divergence of the axial current in a gravitational field background was later performed by Delbourgo and Salam [6], Eguchi and Freund [7] and Delbourgo [8]. As in the ABJ case, these authors found an anomaly associated to the conservation of the axial current, the gravitational axial anomaly. Later, Alvarez-Gaumé and Witten showed the significance of gravitational anomalies for a wide variety of physical applications [9].

The question of absence of radiative corrections to the one loop result obtained for the gravitational axial anomaly is an issue not as well established as it is in the gauge theory case. This is the reason why we proceed to perform an explicit two loop calculation, adopting the spirit in [5]. However, calculating the two loop contribution to the gravitational axial anomaly is a much longer task than to do so for the gauge axial anomaly. In this paper we

shall address the first part of the computation, by calculating the abnormal parity part of the three point function involving one axial vector and two energy-momentum tensors at a specific two loop order in the Abelian Higgs model. The reason we choose to work in this 
model is due to the recent interest arising from the gauge anomaly case in [5], and also due to the fact that this model is a simplified version of the Standard Model. In order to set notation, the anomalous correlator we shall be dealing with is:

$$
\left\langle A_{\alpha}(z) T_{\mu \nu}(y) T_{\rho \sigma}(x)\right\rangle
$$

where $A_{\alpha}$ is the axial current and $T_{\mu \nu}$ the energy-momentum tensor.

The method of calculation [4,5] is based on conformal properties of massless field theories, and also involves ideas from the coordinate space method of differential regularization due to Freedman, Johnson and Latorre [10]. In particular, the correlator (1.1) will be directly calculated in Euclidean position space and a change of variables suggested by the conformal properties of the correlator will be used in order to simplify the internal integrations. The order in two loops we shall be working involves no internal photons, but only internal matter fields (the scalar and spinor fields in the Abelian Higgs model). However, in this case diagrams containing vertex and self-energy corrections will require a regularization scale. To handle this technicality we shall introduce photons in our calculation, as there is a unique choice of gauge fixing parameter (in the photon propagator) which makes both the self-energies and vertex corrections finite. These "finite gauge photons" are merely a technical tool employed in the calculation.

The use of conformal symmetry to construct three point functions is well established. Of particular interest to us is the work by Schreier [11], where three point functions invariant under conformal transformations were constructed. For the case of one axial and two vector currents, it was shown that there is a unique conformal tensor present in the three point function. More recently, Osborn and Petkos [12] and Erdmenger and Osborn [13] have used conformal invariance to compute several three point functions involving the energymomentum tensor. However, the case of one axial current and two energy-momentum tensors was not considered.

What we find in here is that, even though at one loop there is only one conformal tensor present in the correlator (1.1) - the one that leads to the contraction of the Riemann tensor 
with its dual in the expression for the anomaly -, at two loops there are two independent conformal tensors present in the correlator. This is unlike the gauge axial anomaly case where the only possible tensor is the one that leads to the field strength contracted with its dual in the anomaly equation. Precisely because of the presence of these two tensors in the two loop result for the three point function, this correlator does not vanish. Again, this is unlike the gauge axial anomaly case [5].

The two linearly independent conformal tensors present in the anomalous correlator are the ones in expressions (3.6) and (3.7) below (where the notation is explained in the paragraphs leading up to these formulas). One thing we would like to stress is that every diagram relevant for our calculation is either a multiple of one of these tensors, or a linear combination of them both.

Two comments are in order. First, the existence of two independent tensors in the two loop correlator could seem to indicate the existence of a radiative correction to the anomaly. On the other hand, the fact that the correlator does not vanish at two loops does not mean that its divergence (the anomaly) does not vanish at two loops.

Another point of interest is to follow [10] and study the differential regularization of the one loop triangle diagram associated to the gravitational axial anomaly, Figure 1(a). This is done in the Appendix. What one finds is that differential regulation entails the introduction of several different mass scales. Renormalization or symmetry conditions may then be used to determine the ratios of these mass scales. In the gauge axial anomaly case it was found that there is only one mass ratio [10]. In this gravitational axial anomaly case, we have shown in the Appendix that there is more than one mass ratio. This multiplicity of the mass ratios introduces new parameters that could be able to cancel all potential (new) anomalies. Apart from presenting part of these different scales we shall not proceed with their study. Here, we shall only restrict to the calculation of the correlation function, which by itself consists a lengthy project. Extracting the two loop contribution to the gravitational axial anomaly from our three point function is a question we hope to report about in the near future. 
The structure of this paper is as follows. In section 2 we present the massless Abelian Higgs model, as well as a review of the basic ideas involved in the method of calculation we use. This includes the calculation of the one loop triangle diagram. Then, in section 3 we perform our two loop calculation, with emphasis on rigorous details. The many contributing diagrams are organized into separate groups, and then analyzed one at a time.

\section{THE ABELIAN HIGGS MODEL AND CONFORMAL SYMMETRY}

We shall start by presenting the massless Abelian Higgs model. In four dimensional Euclidean space, its action is given by:

$$
S=\int d^{4} x\left\{\frac{1}{4} F_{\mu \nu} F_{\mu \nu}+\left(D_{\mu} \phi\right)^{\dagger} D_{\mu} \phi+\bar{\psi} \gamma_{\mu} D_{\mu} \psi-f \bar{\psi}\left(L \phi+R \phi^{\dagger}\right) \psi-\frac{\lambda}{4}\left(\phi^{\dagger} \phi\right)^{2}\right\},
$$

where we have used $L=\frac{1}{2}\left(1-\gamma_{5}\right)$ and $R=\frac{1}{2}\left(1+\gamma_{5}\right)$, with $\gamma_{5}=\gamma_{1} \gamma_{2} \gamma_{3} \gamma_{4}$. The covariant derivatives are:

$$
\begin{gathered}
D_{\mu} \phi=\left(\partial_{\mu}+i g \mathcal{A}_{\mu}\right) \phi, \\
D_{\mu} \psi=\left(\partial_{\mu}+\frac{1}{2} i g \mathcal{A}_{\mu} \gamma_{5}\right) \psi,
\end{gathered}
$$

so that the theory is parity conserving with pure axial gauge coupling.

Next we introduce a background (external) gravitational field, in order to properly define the energy-momentum tensors associated to the scalar and spinor matter degrees of freedom. A simple way to do this is to couple our model to gravity, so that a spacetime metric $g_{\mu \nu}(x)$ is naturally introduced in the Lagrangian as a field variable. Then we can obtain the energy-momentum tensor by varying the Lagrangian with respect to the metric $g_{\mu \nu}(x)$ as $T_{\mu \nu}(x)=2 \frac{\delta}{\delta g^{\mu \nu}(x)} \int d^{4} x \sqrt{-g} \mathcal{L}$, where $T_{\mu \nu}(x)$ is manifestly symmetric. In addition we have to ensure that it is conserved and traceless, obtaining finally for the fermion field,

$$
T_{\mu \nu}^{\mathbf{F}}=\bar{\psi} \gamma_{(\mu} \partial_{\nu)} \psi
$$

and for the boson field,

$$
T_{\mu \nu}^{\mathrm{B}}=\frac{2}{3}\left\{\partial_{\mu} \phi^{\dagger} \partial_{\nu} \phi+\partial_{\nu} \phi^{\dagger} \partial_{\mu} \phi-\frac{1}{2} \delta_{\mu \nu} \partial_{\alpha} \phi^{\dagger} \partial_{\alpha} \phi-\frac{1}{2}\left(\phi \partial_{\mu} \partial_{\nu} \phi^{\dagger}+\phi^{\dagger} \partial_{\mu} \partial_{\nu} \phi\right)\right\},
$$

where $(\mu \nu) \equiv \mu \nu+\nu \mu$. 
One should observe that in the two loop calculation we are interested in computing the order $\mathcal{O}\left(g f^{2} k^{2}\right)$ correction to the correlator, where $g$ is the gauge coupling, $f$ the scalarspinor coupling, and $k$ the gravitational coupling. This means that there are no internal photons in the associated diagrams, as these would be of order $\mathcal{O}\left(g^{3} k^{2}\right)$ - we shall only need photons as the external axial current, and in order to handle some of the potential divergences in the calculation (see section 3). This is why in (2.3) and (2.4) the scalar and spinor matter degrees of freedom are decoupled from the gauge field.

Conformal symmetry plays a central role in our calculations, as it motivates a change of variables that simplifies the two loop integrations. Due to the absence of any scale, our model is conformal invariant. The conformal group of Euclidean field theory is $O(5,1)$ [12]. All transformations which are continuously connected to the identity are obtained via a combination of rotations and translations with the basic conformal inversion,

$$
\begin{gathered}
x_{\mu}=\frac{x_{\mu}^{\prime}}{x^{\prime 2}} \\
\frac{\partial x_{\mu}}{\partial x_{\nu}^{\prime}}=x^{2}\left(\delta_{\mu \nu}-\frac{2 x_{\mu} x_{\nu}}{x^{2}}\right) \equiv x^{2} J_{\mu \nu}(x) .
\end{gathered}
$$

The Jacobian tensor, $J_{\mu \nu}(x)$, which is an improper orthogonal matrix satisfying $J_{\mu \nu}(x)=$ $J_{\mu \nu}\left(x^{\prime}\right)$, will play a useful role in the calculation of the coordinate space Feynman diagrams.

The action (2.1) is invariant under conformal inversions, as [5]:

$$
\begin{gathered}
\phi(x) \rightarrow \phi^{\prime}(x)=x^{\prime 2} \phi\left(x^{\prime}\right), \\
\psi(x) \rightarrow \psi^{\prime}(x)={x^{\prime}}^{2} \gamma_{5} \not^{\prime} \psi\left(x^{\prime}\right), \\
\mathcal{A}_{\mu}(x) \rightarrow \mathcal{A}_{\mu}^{\prime}(x)=-x^{\prime 2} J_{\mu \nu}\left(x^{\prime}\right) \mathcal{A}_{\nu}\left(x^{\prime}\right),
\end{gathered}
$$

while also the following relations hold,

$$
d^{4} x=\frac{d^{4} x^{\prime}}{x^{\prime 8}} \quad \text { and } \quad \not x^{\prime} \gamma_{\mu} x^{\prime}=-x^{\prime 2} J_{\mu \nu}\left(x^{\prime}\right) \gamma_{\nu}
$$

In order to use conformal properties to simplify the two loop Feynman integrals, one should expect that the relevant Feynman rules will consist of vertex factors and propagators 
with simple inversion properties. In particular for the scalar and spinor propagators we have,

$$
\begin{gathered}
\Delta(x-y)=\frac{1}{4 \pi^{2}} \frac{1}{(x-y)^{2}}=\frac{1}{4 \pi^{2}} \frac{x^{\prime 2} y^{\prime 2}}{\left(x^{\prime}-y^{\prime}\right)^{2}}, \\
S(x-y)=-\not \partial \Delta(x-y)=\frac{1}{2 \pi^{2}} \frac{\not x-y}{(x-y)^{4}}=-\frac{1}{2 \pi^{2}} x^{\prime 2} y^{\prime 2} \not \not^{\prime} \frac{\not x^{\prime}-y^{\prime}}{\left(x^{\prime}-y^{\prime}\right)^{4}} y^{\prime} .
\end{gathered}
$$

The vertex rules, read from the action (2.1), are:

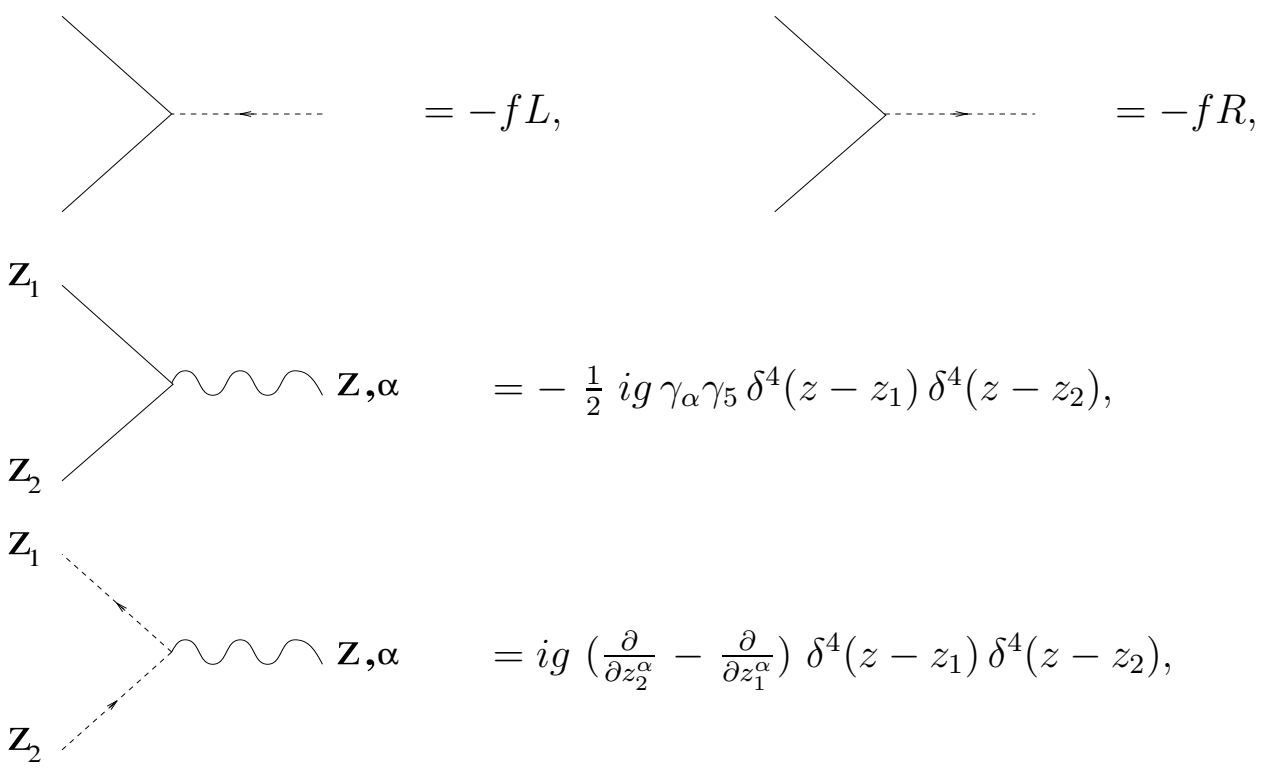

where solid lines are fermions, dashed lines are scalars and wavy lines are gauge fields.

In addition the energy-momentum tensor insertions (2.3) and (2.4) yield the following vertices:

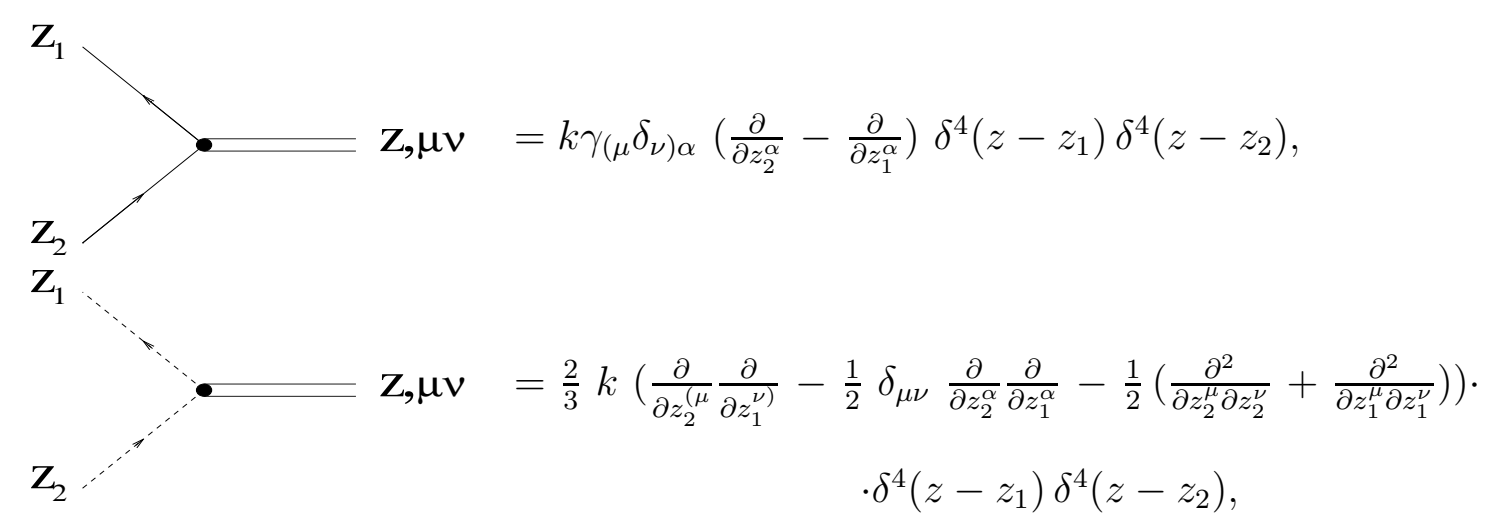


where the double solid lines represent gravitons.

Let us analyze the conformal properties of the graviton vertices. In order to do that, we attach the vertices (2.12) and (2.13) to scalar and spinor legs, and use (2.5), (2.7) and (2.8) to obtain,

$$
\begin{gathered}
S(v-x) \gamma_{(\mu} \delta_{\nu) \alpha}\left(\frac{\vec{\partial}}{\partial x_{\alpha}}-\frac{\overleftarrow{\partial}}{\partial x_{\alpha}}\right) S(x-u)= \\
=-x^{\prime 8} J_{\bar{\mu}(\mu}\left(x^{\prime}\right) J_{\nu) \bar{\nu}}\left(x^{\prime}\right)\left\{v^{\prime 2} \psi^{\prime} S\left(v^{\prime}-x^{\prime}\right) \gamma_{\bar{\mu}}\left(\frac{\vec{\partial}}{\partial x_{\bar{\nu}}^{\prime}}-\frac{\overleftarrow{\partial}}{\partial x_{\bar{\nu}}^{\prime}}\right) S\left(x^{\prime}-u^{\prime}\right) u^{\prime 2} \psi^{\prime}\right\}
\end{gathered}
$$

for the fermionic vertex, where the derivatives only act inside the curly brackets. Likewise,

$$
\begin{gathered}
\Delta(v-x)\left(\frac{\overleftarrow{\partial}}{\partial x_{(\mu}} \frac{\vec{\partial}}{\left.\partial x_{\nu}\right)}-\frac{1}{2} \delta_{\mu \nu} \frac{\overleftarrow{\partial}}{\partial x_{\alpha}} \frac{\vec{\partial}}{\partial x_{\alpha}}-\frac{1}{2}\left(\frac{\overleftarrow{\partial^{2}}}{\partial x_{\mu} \partial x_{\nu}}+\frac{\overrightarrow{\partial^{2}}}{\partial x_{\mu} \partial x_{\nu}}\right)\right) \Delta(x-u)=x^{\prime 8} J_{\bar{\mu} \mu}\left(x^{\prime}\right) J_{\nu \bar{\nu}}\left(x^{\prime}\right) \cdot \\
\cdot\left\{v^{\prime 2} \Delta\left(v^{\prime}-x^{\prime}\right)\left(\frac{\overleftarrow{\partial}}{\partial x_{(\bar{\mu}}^{\prime}} \frac{\vec{\partial}}{\partial x_{\bar{\nu})}^{\prime}}-\frac{1}{2} \delta_{\bar{\mu} \bar{\nu}} \frac{\overleftarrow{\partial}}{\partial x_{\alpha}^{\prime}} \frac{\vec{\partial}}{\partial x_{\alpha}^{\prime}}-\frac{1}{2}\left(\frac{\overleftarrow{\partial^{2}}}{\partial x_{\bar{\mu}}^{\prime} \partial x_{\bar{\nu}}^{\prime}}+\frac{\overrightarrow{\partial^{2}}}{\partial x_{\bar{\mu}}^{\prime} \partial x_{\bar{\nu}}^{\prime}}\right)\right) \Delta\left(x^{\prime}-u^{\prime}\right) u^{\prime 2}\right\}, \quad(2.15)
\end{gathered}
$$

for the bosonic vertex, where once again the derivatives only act inside the curly brackets.

As an illustration of these coordinate space propagators and vertex rules, we shall now look at the one loop triangle diagram and perform the conformal inversion on the amplitude's tensor structure. The relevant one loop triangle diagram is depicted in Figure 1(a) and its amplitude, $B_{\alpha, \mu \nu, \rho \sigma}(z, y, x)$, can be computed using the previous rules to be:

$$
\begin{gathered}
B_{\alpha, \mu \nu, \rho \sigma}(z, y, x)= \\
=\frac{1}{2} i g k^{2} \operatorname{Tr} \gamma_{\alpha} \gamma_{5} S(z-y) \gamma_{(\mu} \delta_{\nu) \beta}\left(\frac{\vec{\partial}}{\partial y_{\beta}}-\frac{\overleftarrow{\partial}}{\partial y_{\beta}}\right) S(y-x) \gamma_{(\rho} \delta_{\sigma) \pi}\left(\frac{\vec{\partial}}{\partial x_{\pi}}-\frac{\overleftarrow{\partial}}{\partial x_{\pi}}\right) S(x-z)
\end{gathered}
$$

Due to translation symmetry we are free to set $z=0$, while we refer the remaining external points $x$ and $y$ to their inverted images (2.5). Although this transformation may seem $a d$ hoc at this stage, it will later simplify the calculation of the two loop diagrams [5]. The result we obtain is,

$$
B_{\alpha, \mu \nu, \rho \sigma}(0, y, x)=-\frac{i g k^{2}}{8 \pi^{4}} y^{\prime 8} x^{\prime 8} J_{\bar{\mu}(\mu}\left(y^{\prime}\right) J_{\nu) \bar{\nu}}\left(y^{\prime}\right) J_{\bar{\rho}(\rho}\left(x^{\prime}\right) J_{\sigma) \bar{\sigma}}\left(x^{\prime}\right) \operatorname{Tr} \gamma_{\alpha} \gamma_{5} \gamma_{\bar{\mu}} \frac{\partial^{2}}{\partial y_{\bar{\nu}}^{\prime} \partial x_{\bar{\sigma}}^{\prime}} S\left(y^{\prime}-x^{\prime}\right) \gamma_{\bar{\rho}} .
$$

Taking the fermionic trace one finally gets,

$$
B_{\alpha, \mu \nu, \rho \sigma}(0, y, x)=\frac{i g k^{2}}{4 \pi^{6}} y^{\prime 8} x^{\prime 8} J_{\bar{\mu}(\mu}\left(y^{\prime}\right) J_{\nu) \bar{\nu}}\left(y^{\prime}\right) J_{\bar{\rho}(\rho}\left(x^{\prime}\right) J_{\sigma) \bar{\sigma}}\left(x^{\prime}\right) \varepsilon_{\alpha \kappa \bar{\mu} \bar{\rho}} \frac{\partial^{2}}{\partial y_{\bar{\nu}}^{\prime} \partial x_{\bar{\sigma}}^{\prime}} \frac{\left(x^{\prime}-y^{\prime}\right)_{\kappa}}{\left(x^{\prime}-y^{\prime}\right)^{4}} .
$$


For separated points (2.16) is fully Bose symmetric and conserved on all indices. The expected anomaly is a local violation of the conservation Ward identities which arises because the differentiation of singular functions is involved [5]. There are several ways to obtain the anomaly in this coordinate space approach [10, [14, 5]. One way [10,5] to do this is to recognize that the amplitude (2.16) is too singular at short distances to have a well defined Fourier transform. One then regulates, which entails the introduction of several independent mass scales. The regulated amplitude is well defined, and one can check the Ward identities. Also, an important aspect of this coordinate space approach to the axial anomaly is that the well defined amplitude (2.16), for separated points, determines the fact that there is an anomaly of specific strength [10].

Some more comments about the role of the conformal symmetry in the calculation of possible radiative corrections to the anomaly are now in order. At first sight this could look as a questionable role, after all the introduction of a scale to handle the divergences of perturbation theory will spoil any expected conformal properties. This is true in general, but our two loop triangle diagrams for this massless Abelian Higgs model are exceptional. Any primitively divergent amplitude is exceptional when studied in coordinate space for separated points, since the internal integrals converge without regularization [5].

As we shall see in the next section, there will be 3 non-planar and 3 planar diagrams, which are primitives. Of course there will be many other diagrams which contain subdivergent vertex and self-energy corrections, and these require a regularization scale. However, we are dealing with pure axial coupling for the fermion. This means that if we introduce diagrams containing internal photons, then there is a unique choice of gauge-fixing parameter $\Gamma$ which makes the one loop self-energy finite [5]. Moreover, since the vertex and self-energy corrections are related by a Ward identity, each vertex correction is also finite in this same gauge. In conclusion, choosing this finite gauge makes it possible to obtain a finite two loop result in our calculation. 


\section{THE THREE POINT FUNCTION FOR THE TWO LOOP GRAVITATIONAL AXIAL ANOMALY}

Let us now proceed to the next loop order in the non-gauge sector, as we are interested in computing possible corrections to the gravitational axial anomaly at order $\mathcal{O}\left(g f^{2} k^{2}\right)$. At this order we have a total of 36 diagrams that can possibly contribute. Of these diagrams, 3 are non-planar, but they actually only correspond to 2 independent calculations due to reflection symmetry. These are depicted in Figures 1(b) and 1(c). Then, there are 3 scalar self-energy diagrams, and other 3 photon self-energy diagrams (as we shall see, some diagrams involving photons are required in order to choose the finite gauge and compensate some divergences of the non-gauge amplitudes). These 6 self-energy diagrams amount to 2 independent calculations alone, the ones depicted in Figures 1(d) and 1(e). Then, we have 3 axial current insertion vertex corrections, in Figures $1(\mathrm{f}), 1(\mathrm{~g})$ and $1(\mathrm{~h})$. At the energy-momentum tensor insertion, we also have vertex corrections. These are 6 diagrams, amounting to the 3 independent calculations in Figures 1(i), 1(j) and 1(k). There are also 6 diagrams that identically vanish due to fermionic traces, the ones in Figures $1(1), 1(\mathrm{~m})$ and 1(n). Associated to the mentioned self-energies there are 3 diagrams corresponding to local self-energy renormalizations. They amount to 1 independent calculation, Figure 1(o). Also, associated to the mentioned vertex corrections at the energy-momentum insertion there are 2 diagrams corresponding to local vertex renormalizations. They amount to 1 independent calculation, Figure 1(p). So, overall, of the 29 initial two loop diagrams in Figure 1, we are left with 12 independent calculations. In Figure 2 we have 7 more diagrams, corresponding to 4 independent calculations. These diagrams are associated to the finite gauge photons and shall be discussed later. We are thus left with an overall number of 16 independent calculations, out of the initial 36 diagrams. Let us see how to perform such calculations, one at a time. 


\section{A. Diagrams in Figures 1(b) and 1(c)}

We begin with the non-planar diagram depicted in Figure 1(b), which we shall denote by $N_{\alpha, \mu \nu, \rho \sigma}^{(1)}(z, y, x)$. This amplitude is conformal covariant since no issues of subdivergences and gauge choice arise. The idea [⿴囗十 is to use the inversion, $u_{\alpha}=u_{\alpha}^{\prime} / u^{\prime 2}$ and $v_{\alpha}=v^{\prime}{ }_{\alpha} / v^{\prime 2}$, as a change of variables in the internal integrals. In order to use the simple conformal properties of the propagators (2.8) we must also refer the external points to their inverted images (2.5), as was done in $(2.14),(2.15)$, and in $(2.17),(2.18)$. If in succession we use the translation symmetry to place one point at the origin, say $z=0$, then the propagators attached to that point drop out of the integral, because the inverted point is now at $\infty$, and the integrals simplify.

After summing over both directions of Higgs field propagation, and setting $z=0$, the amplitude for $N_{\alpha, \mu \nu, \rho \sigma}^{(1)}(0, y, x)$ is written as,

$$
\begin{gathered}
N_{\alpha, \mu \nu, \rho \sigma}^{(1)}(0, y, x)=-\frac{i g f^{2} k^{2}}{8 \pi^{4}} \int d^{4} u d^{4} v\left(\frac{u_{\alpha}}{u^{4} v^{2}}-\frac{v_{\alpha}}{v^{4} u^{2}}\right) \\
\cdot \operatorname{Tr} \gamma_{5} S(v-x) \gamma_{(\rho} \delta_{\sigma) \beta}\left(\frac{\vec{\partial}}{\partial x_{\beta}}-\frac{\overleftarrow{\partial}}{\partial x_{\beta}}\right) S(x-u) S(u-y) \gamma_{(\mu} \delta_{\nu) \pi}\left(\frac{\vec{\partial}}{\partial y_{\pi}}-\frac{\overleftarrow{\partial}}{\partial y_{\pi}}\right) S(y-v)
\end{gathered}
$$

The change of variables previously outlined can be performed with the help of $(2.7),(2.8)$, and the Higgs current transformation,

$$
\frac{u_{\alpha}}{u^{4} v^{2}}-\frac{v_{\alpha}}{v^{4} u^{2}}=v^{\prime 2} u^{\prime 2}\left(u_{\alpha}^{\prime}-v_{\alpha}^{\prime}\right)
$$

The spinor propagator side factors $\not^{\prime}, \psi^{\prime}$, etc., collapse within the trace, and the Jacobian $\left(u^{\prime} v^{\prime}\right)^{-8}$ cancels with factors in the numerator. Performing the algebra we obtain,

$$
\begin{gathered}
N_{\alpha, \mu \nu, \rho \sigma}^{(1)}(0, y, x)=-\frac{i g f^{2} k^{2}}{8 \pi^{4}} y^{\prime 8} x^{\prime 8} J_{\bar{\mu}(\mu}\left(y^{\prime}\right) J_{\nu) \bar{\nu}}\left(y^{\prime}\right) J_{\bar{\rho}(\rho}\left(x^{\prime}\right) J_{\sigma) \bar{\sigma}}\left(x^{\prime}\right) \int d^{4} u^{\prime} d^{4} v^{\prime}\left(v^{\prime}-u^{\prime}\right)_{\alpha} \cdot \\
\cdot\left\{\operatorname{Tr} \gamma_{5} S\left(v^{\prime}-x^{\prime}\right) \gamma_{\bar{\rho}}\left(\frac{\vec{\partial}}{\partial x_{\bar{\sigma}}^{\prime}}-\frac{\overleftarrow{\partial}}{\partial x_{\bar{\sigma}}^{\prime}}\right) S\left(x^{\prime}-u^{\prime}\right) S\left(u^{\prime}-y^{\prime}\right) \gamma_{\bar{\mu}}\left(\frac{\vec{\partial}}{\partial y_{\bar{\nu}}^{\prime}}-\frac{\overleftarrow{\partial}}{\partial y_{\bar{\nu}}^{\prime}}\right) S\left(y^{\prime}-v^{\prime}\right)\right\}, \quad
\end{gathered}
$$

where the derivatives are acting only inside the curly brackets.

We see that we obtain the expected transformation factors for the energy-momentum tensors at $x$ and $y$ times an integral in which $u^{\prime}$ and $v^{\prime}$ each appear in only two denominators. 
These convolution integrals can be done in several different ways. The final relevant formulas are listed in the Appendix. We begin by using the trace properties to move the $S\left(y^{\prime}-v^{\prime}\right)$ propagator in $(3.3)$ close to the $S\left(v^{\prime}-x^{\prime}\right)$ propagator. The differential operators are kept fixed, with the understanding that now the $y^{\prime}$ derivative that seems to be acting on nothing is actually acting on the propagator $S\left(y^{\prime}-v^{\prime}\right)$ which is now sitting on the left. As usual, all derivatives act only inside curly brackets. We can perform the integrations without the need to make the differentiations first as the integration variables are well separated from the differentiation ones. Expand the product with $\left(v^{\prime}-u^{\prime}\right)_{\alpha}$, and we are led to the following result:

$$
\begin{aligned}
& \int d^{4} u^{\prime} d^{4} v^{\prime}\left(v^{\prime}-u^{\prime}\right)_{\alpha} \operatorname{Tr} \gamma_{5} S\left(v^{\prime}-x^{\prime}\right) \gamma_{\bar{\rho}}\left(\frac{\vec{\partial}}{\partial x_{\bar{\sigma}}^{\prime}}-\frac{\overleftarrow{\partial}}{\partial x_{\bar{\sigma}}^{\prime}}\right) S\left(x^{\prime}-u^{\prime}\right) S\left(u^{\prime}-y^{\prime}\right) \gamma_{\bar{\mu}}\left(\frac{\vec{\partial}}{\partial y_{\bar{\nu}}^{\prime}}-\frac{\overleftarrow{\partial}}{\partial y_{\bar{\nu}}^{\prime}}\right) S\left(y^{\prime}-v^{\prime}\right)= \\
& -\operatorname{Tr} \gamma_{5} \int d^{4} v^{\prime} v_{\alpha}^{\prime} S\left(v^{\prime}-y^{\prime}\right) S\left(v^{\prime}-x^{\prime}\right) \gamma_{\bar{\rho}}\left(\frac{\vec{\partial}}{\partial x_{\bar{\sigma}}^{\prime}}-\frac{\overleftarrow{\partial}}{\partial x_{\bar{\sigma}}^{\prime}}\right) \int d^{4} u^{\prime} S\left(u^{\prime}-x^{\prime}\right) S\left(u^{\prime}-y^{\prime}\right) \gamma_{\bar{\mu}}\left(\frac{\vec{\partial}}{\partial y_{\bar{\nu}}^{\prime}}-\frac{\overleftarrow{\partial}}{\partial y_{\bar{\nu}}^{\prime}}\right) \\
& +\operatorname{Tr} \gamma_{5} \int d^{4} v^{\prime} S\left(v^{\prime}-y^{\prime}\right) S\left(v^{\prime}-x^{\prime}\right) \gamma_{\bar{\rho}}\left(\frac{\vec{\partial}}{\partial x_{\bar{\sigma}}^{\prime}}-\frac{\overleftarrow{\partial}}{\partial x_{\bar{\sigma}}^{\prime}}\right) \int d^{4} u^{\prime} u_{\alpha}^{\prime} S\left(u^{\prime}-x^{\prime}\right) S\left(u^{\prime}-y^{\prime}\right) \gamma_{\bar{\mu}}\left(\frac{\vec{\partial}}{\partial y_{\bar{\nu}}^{\prime}}-\frac{\overleftarrow{\partial}}{\partial y_{\bar{\nu}}^{\prime}}\right)
\end{aligned}
$$

where the integrals can be directly read off from the Appendix. When these results are used and substituted within the trace, one finds the final amplitude,

$$
\begin{gathered}
N_{\alpha, \mu \nu, \rho \sigma}^{(1)}(0, y, x)=-\frac{i g f^{2} k^{2}}{32 \pi^{8}} y^{\prime 8} x^{\prime 8} J_{\bar{\mu}(\mu}\left(y^{\prime}\right) J_{\nu) \bar{\nu}}\left(y^{\prime}\right) J_{\bar{\rho}(\rho}\left(x^{\prime}\right) J_{\sigma) \bar{\sigma}}\left(x^{\prime}\right) \cdot \\
\cdot\left\{\varepsilon_{\alpha \kappa \bar{\rho} \bar{\mu}} \frac{1}{\left(x^{\prime}-y^{\prime}\right)^{2}}\left(\frac{\vec{\partial}}{\partial x^{\prime} \bar{\sigma}}-\frac{\overleftarrow{\partial}}{\partial x_{\bar{\sigma}}^{\prime}}\right) \frac{\left(x^{\prime}-y^{\prime}\right)_{\kappa}}{\left(x^{\prime}-y^{\prime}\right)^{2}}\left(\frac{\vec{\partial}}{\partial y_{\bar{\nu}}^{\prime}}-\frac{\overleftarrow{\partial}}{\partial y_{\bar{\nu}}^{\prime}}\right)\right\},
\end{gathered}
$$

where we should have the "trace" attitude for taking derivatives: the derivatives only act inside the curly brackets, and the $y^{\prime}$ derivative that seems to be acting on nothing is actually acting on the first $\left(x^{\prime}-y^{\prime}\right)$ term.

We observe that unlike the case in [5], this non-planar amplitude is not a numerical multiple of the amplitude for the one loop triangle diagram (2.18). This is because the tensor (derivative) structure in (3.5) is different from the one in (2.18). To see that one just has to explicitly compute both structures, and compare them. For the triangle one has:

$$
\frac{\partial^{2}}{\partial x_{\bar{\sigma}}^{\prime} \partial y_{\bar{\nu}}^{\prime}} \frac{\Delta_{\kappa}}{\Delta^{4}}=
$$




$$
=\frac{\partial}{\partial x_{\bar{\sigma}}^{\prime}}\left(\frac{\Delta_{\kappa}}{\Delta^{2}}\right) \frac{\partial}{\partial y_{\bar{\nu}}^{\prime}}\left(\frac{1}{\Delta^{2}}\right)+\frac{\Delta_{\kappa}}{\Delta^{2}} \frac{\partial^{2}}{\partial x_{\bar{\sigma}}^{\prime} \partial y_{\bar{\nu}}^{\prime}}\left(\frac{1}{\Delta^{2}}\right)+\frac{1}{\Delta^{2}} \frac{\partial^{2}}{\partial x_{\bar{\sigma}}^{\prime} \partial y_{\bar{\nu}}^{\prime}}\left(\frac{\Delta_{\kappa}}{\Delta^{2}}\right)+\frac{\partial}{\partial x_{\bar{\sigma}}^{\prime}}\left(\frac{1}{\Delta^{2}}\right) \frac{\partial}{\partial y_{\bar{\nu}}^{\prime}}\left(\frac{\Delta_{\kappa}}{\Delta^{2}}\right),
$$

while for the non-planar structure one obtains,

$$
\begin{gathered}
\frac{1}{\Delta^{2}}\left(\frac{\vec{\partial}}{\partial x^{\prime}{ }_{\bar{\sigma}}}-\frac{\overleftarrow{\partial}}{\partial x^{\prime}{ }_{\bar{\sigma}}}\right) \frac{\Delta_{\kappa}}{\Delta^{2}}\left(\frac{\vec{\partial}}{\partial y_{\bar{\nu}}^{\prime}}-\frac{\overleftarrow{\partial}}{\partial y_{\bar{\nu}}^{\prime}}\right)= \\
=\frac{\partial}{\partial x_{\bar{\sigma}}^{\prime}}\left(\frac{\Delta_{\kappa}}{\Delta^{2}}\right) \frac{\partial}{\partial y_{\bar{\nu}}^{\prime}}\left(\frac{1}{\Delta^{2}}\right)-\frac{\Delta_{\kappa}}{\Delta^{2}} \frac{\partial^{2}}{\partial x_{\bar{\sigma}}^{\prime} \partial y_{\bar{\nu}}^{\prime}}\left(\frac{1}{\Delta^{2}}\right)-\frac{1}{\Delta^{2}} \frac{\partial^{2}}{\partial x_{\bar{\sigma}}^{\prime} \partial y_{\bar{\nu}}^{\prime}}\left(\frac{\Delta_{\kappa}}{\Delta^{2}}\right)+\frac{\partial}{\partial x_{\bar{\sigma}}^{\prime}}\left(\frac{1}{\Delta^{2}}\right) \frac{\partial}{\partial y_{\bar{\nu}}^{\prime}}\left(\frac{\Delta_{\kappa}}{\Delta^{2}}\right)
\end{gathered}
$$

where we defined $\Delta \equiv\left(x^{\prime}-y^{\prime}\right)$. The reason such difference can happen is that while in [5] there is a unique conformal tensor structure for the correlator of three axial vector currents, in here we have two conformal tensor structures due to the higher dimensionality of the correlator of the one axial vector current and the two energy-momentum tensors. Also, observe that both these structures (3.6) and (3.7) are to be understood as always attached to the appropriate factors of $J_{\mu \nu}\left(y^{\prime}\right), J_{\rho \sigma}\left(x^{\prime}\right)$ and the appropriate powers of $y^{\prime}, x^{\prime}$. Moreover the diagrams that give rise to them obey conservation equations for the energy-momentum tensor insertions. (3.6) is associated to the one loop diagram in (2.18). It can easily be proved that the conservation equation is obeyed, a standard result from [6 8] (and also form [10] once we are aware of the relation (A.6) from the Appendix). (3.7) is associated to the two loop diagram in (3.5), and one can also explicitly check the conservation law for this case. This existence of two conformal structures is an extra feature in the discussion of these two loop diagrams, relative to the work in [5].

There are 2 more non-planar diagrams, where the scalar vertex is placed at $x$ and at $y$. We need to compute them, as they are independent of the previous result (we have a scalarscalar-tensor vertex instead of a scalar-scalar-vector vertex, among other different vertices), but they amount to 1 independent calculation.

So, we proceed with the non-planar diagram in Figure 1(c), denoted in the following by $N_{\alpha, \mu \nu, \rho \sigma}^{(2)}(z, y, x)$. The method of calculation is very similar to the one for the previous diagram, and so we shall perform it in here with somewhat less details. After summing over both directions of Higgs field propagation, and setting $z=0$, the amplitude for $N_{\alpha, \mu \nu, \rho \sigma}^{(2)}(0, y, x)$ is 
written as,

$$
\begin{gathered}
N_{\alpha, \mu \nu, \rho \sigma}^{(2)}(0, y, x)=-\frac{i g f^{2} k^{2}}{12 \pi^{4}} \int d^{4} u d^{4} v \operatorname{Tr} \gamma_{5} \frac{\psi \gamma_{\alpha} \psi}{u^{4} v^{4}} S(v-x) \gamma_{(\rho} \delta_{\sigma) \beta}\left(\frac{\vec{\partial}}{\partial x_{\beta}}-\frac{\overleftarrow{\partial}}{\partial x_{\beta}}\right) S(x-u) \\
\cdot \Delta(u-y)\left(\frac{\overleftarrow{\partial}}{\partial y_{(\mu}} \frac{\vec{\partial}}{\left.\partial y_{\nu}\right)}-\frac{1}{2} \delta_{\mu \nu} \frac{\overleftarrow{\partial}}{\partial y_{\pi}} \frac{\vec{\partial}}{\partial y_{\pi}}-\frac{1}{2}\left(\frac{\overleftarrow{\partial^{2}}}{\partial y_{\mu} \partial y_{\nu}}+\frac{\overrightarrow{\partial^{2}}}{\partial y_{\mu} \partial y_{\nu}}\right)\right) \Delta(y-v)
\end{gathered}
$$

Performing the conformal inversion is now no harder than it was for the previous diagram. The procedure is essentially the same, and if we carry out the algebra we obtain,

$$
\begin{gathered}
N_{\alpha, \mu \nu, \rho \sigma}^{(2)}(0, y, x)=-\frac{i g f^{2} k^{2}}{24 \pi^{4}} y^{\prime 8} x^{\prime 8} J_{\bar{\mu}(\mu}\left(y^{\prime}\right) J_{\nu) \bar{\nu}}\left(y^{\prime}\right) J_{\bar{\rho}(\rho}\left(x^{\prime}\right) J_{\sigma) \bar{\sigma}}\left(x^{\prime}\right) \cdot \\
\cdot \int d^{4} u^{\prime} d^{4} v^{\prime}\left\{\operatorname{Tr} \gamma_{5} \gamma_{\alpha} S\left(v^{\prime}-x^{\prime}\right) \gamma_{\bar{\rho}}\left(\frac{\vec{\partial}}{\partial x_{\bar{\sigma}}^{\prime}}-\frac{\overleftarrow{\partial}}{\partial x_{\bar{\sigma}}^{\prime}}\right) S\left(x^{\prime}-u^{\prime}\right) \cdot\right. \\
\left.\cdot \Delta\left(u^{\prime}-y^{\prime}\right)\left(\frac{\overleftarrow{\partial}}{\partial y^{\prime}{ }_{(\bar{\mu}}} \frac{\vec{\partial}}{\left.\partial y_{\bar{\nu}}^{\prime}\right)}-\frac{1}{2} \delta_{\bar{\mu} \bar{\nu}} \frac{\overleftarrow{\partial}}{\partial y_{\pi}^{\prime}} \frac{\vec{\partial}}{\partial y_{\pi}^{\prime}}-\frac{1}{2}\left(\frac{\grave{\partial}^{2}}{\partial y_{\bar{\mu}}^{\prime} \partial y_{\bar{\nu}}^{\prime}}+\frac{\partial^{2}}{\partial y_{\bar{\mu}}^{\prime} \partial y_{\bar{\nu}}^{\prime}}\right)\right) \Delta\left(y^{\prime}-v^{\prime}\right)\right\}
\end{gathered}
$$

Once again the expected structure emerges, and all we have to do is to perform the integrations. Using the relevant formulas from the Appendix, we find the final result as,

$$
\begin{gathered}
N_{\alpha, \mu \nu, \rho \sigma}^{(2)}(0, y, x)=\frac{i g f^{2} k^{2}}{384 \pi^{8}} y^{\prime 8} x^{\prime 8} J_{\bar{\mu}(\mu}\left(y^{\prime}\right) J_{\nu) \bar{\nu}}\left(y^{\prime}\right) J_{\bar{\rho}(\rho}\left(x^{\prime}\right) J_{\sigma) \bar{\sigma}}\left(x^{\prime}\right)\left\{\varepsilon_{\alpha \ell \bar{\rho} \kappa} \cdot\right. \\
\left.\cdot \frac{\left(x^{\prime}-y^{\prime}\right)_{\ell}}{\left(x^{\prime}-y^{\prime}\right)^{2}}\left(\frac{\vec{\partial}}{\partial x_{\bar{\sigma}}^{\prime}}-\frac{\overleftarrow{\partial}}{\partial x_{\bar{\sigma}}^{\prime}}\right) \frac{\left(x^{\prime}-y^{\prime}\right)_{\kappa}}{\left(x^{\prime}-y^{\prime}\right)^{2}}\left(\frac{\overleftarrow{\partial}}{\partial y_{(\bar{\mu}}^{\prime}} \frac{\vec{\partial}}{\partial y_{\bar{\nu})}^{\prime}}-\frac{1}{2} \delta_{\bar{\mu} \bar{\nu}} \frac{\overleftarrow{\partial}}{\partial y_{\pi}^{\prime}} \frac{\vec{\partial}}{\partial y_{\pi}^{\prime}}-\frac{1}{2}\left(\frac{\overleftarrow{\partial^{2}}}{\partial y_{\bar{\mu}}^{\prime} \partial y_{\bar{\nu}}^{\prime}}+\frac{\overrightarrow{\partial^{2}}}{\partial y_{\bar{\mu}}^{\prime} \partial y_{\bar{\nu}}^{\prime}}\right)\right)\right\} .
\end{gathered}
$$

However, one should note the following. In (3.3) both differential operators were first order in the derivatives, but in (3.9) the differential operator associated to the vertex (2.13) is actually second order. As we expect to have at the end a result similar to (3.5) or (2.18), we have to perform one of the derivatives in order for both differential operators to become first order. Manipulating this result through a somewhat lengthy calculation, one finds:

$$
\begin{gathered}
N_{\alpha, \mu \nu, \rho \sigma}^{(2)}(0, y, x)=-\frac{i g f^{2} k^{2}}{128 \pi^{8}} y^{\prime 8} x^{\prime 8} J_{\bar{\mu}(\mu}\left(y^{\prime}\right) J_{\nu) \bar{\nu}}\left(y^{\prime}\right) J_{\bar{\rho}(\rho}\left(x^{\prime}\right) J_{\sigma) \bar{\sigma}}\left(x^{\prime}\right) . \\
\cdot\left\{\varepsilon_{\alpha \kappa \bar{\rho} \bar{\mu}} \frac{1}{\left(x^{\prime}-y^{\prime}\right)^{2}}\left(\frac{\vec{\partial}}{\partial x^{\prime} \bar{\sigma}}-\frac{\overleftarrow{\partial}}{\partial x_{\bar{\sigma}}^{\prime}}\right) \frac{\left(x^{\prime}-y^{\prime}\right)_{\kappa}}{\left(x^{\prime}-y^{\prime}\right)^{2}}\left(\frac{\vec{\partial}}{\partial y_{\bar{\nu}}^{\prime}}-\frac{\overleftarrow{\partial}}{\partial y_{\bar{\nu}}^{\prime}}\right)\right\},
\end{gathered}
$$

where the notation is like in (3.5). One realizes that the structure here obtained is the same as in (3.5). So, the 3 non-planar diagrams have the same tensor structure, which is 
different from the one associated to the one loop triangle. From this result we immediately read the last non-planar diagram, the one with the vertex involving the scalar fields and energy-momentum tensor located at $x$. All we have to do is to exchange $x$ with $y$ and $\mu \nu$ with $\rho \sigma$ in (3.11). This actually does not change the amplitude (3.11), so that this third diagram contributes with the same amount as its reflection symmetric diagram.

Finally, we can add these 3 diagrams, and obtain the non-planar contribution to the two loop correlator. The overall contribution is simply:

$$
\begin{gathered}
N_{\alpha, \mu \nu, \rho \sigma}(0, y, x)=\sum_{i=1}^{3} N_{\alpha, \mu \nu, \rho \sigma}^{(i)}(0, y, x)=-\frac{3 i g f^{2} k^{2}}{64 \pi^{8}} y^{\prime 8} x^{\prime 8} J_{\bar{\mu}(\mu}\left(y^{\prime}\right) J_{\nu) \bar{\nu}}\left(y^{\prime}\right) J_{\bar{\rho}(\rho}\left(x^{\prime}\right) J_{\sigma) \bar{\sigma}}\left(x^{\prime}\right) \\
\cdot\left\{\varepsilon_{\alpha \kappa \bar{\rho} \bar{\mu}} \frac{1}{\left(x^{\prime}-y^{\prime}\right)^{2}}\left(\frac{\vec{\partial}}{\partial x^{\prime} \bar{\sigma}}-\frac{\overleftarrow{\partial}}{\partial x^{\prime} \bar{\sigma}}\right) \frac{\left(x^{\prime}-y^{\prime}\right)_{\kappa}}{\left(x^{\prime}-y^{\prime}\right)^{2}}\left(\frac{\vec{\partial}}{\partial y_{\bar{\nu}}^{\prime}}-\frac{\overleftarrow{\partial}}{\partial y_{\bar{\nu}}^{\prime}}\right)\right\}
\end{gathered}
$$

\section{B. Diagrams in Figures 1(d), 1(e) and 1(o)}

We now proceed to the self-energy diagrams. These will be the same as in the three gauge current case [5]. We shall see the finite gauge mechanism for the one loop selfenergies and vertex corrections coming about, as it handles certain divergences by choosing a gauge where they are zero [4,5]. For this cancelation of divergences we have introduced the Abelian field which can be decoupled at the end by setting its coupling to zero. Let us see how all that works, by starting with the Higgs self-energy diagram in Figure 1(d), and the photon self-energy diagram in Figure 1(e). These are 3 diagrams in Figure 1(d) (as we can place the self-energy loop at any of the 3 sides of the triangle), which amount to 1 independent calculation, and other 3 diagrams in Figure 1(e) that again amount to 1 independent calculation. If we remove the self-energy leg from the triangle diagram, and add the Higgs and photon contributions we obtain [5],

$$
\Sigma(v-u)=\frac{1}{8 \pi^{4}}\left[f^{2}+\frac{1}{2} g^{2}(1-\Gamma)\right] \frac{\psi-\not}{(v-u)^{6}}+a \not \partial \delta^{4}(v-u),
$$

where $\Gamma$ is the gauge fixing parameter coming from the photon propagator [4.5]. In this

result, the first term is the part of the amplitude which is determined by the Feynman rules 
read from the diagrams. It has a linearly divergent Fourier transform, but the crucial point is that this amplitude can be made finite by choosing the gauge $\Gamma=1+2 f^{2} / g^{2}$. It then vanishes for separated points. However, there is a possible local term, the second term in (3.13), which is left ambiguous by the Feynman rules, and is represented in Figure 1(o). The constant $a$ will be determined by the Ward identity [5].

In order to proceed with the calculation of this constant using the Ward identity, we first need to look at the following vertex correction diagrams at the axial current insertion: Figure 1(f), $T_{\alpha, \mu \nu, \rho \sigma}^{(1)}(z, y, x)$, Figure 1(g), $T_{\alpha, \mu \nu, \rho \sigma}^{(2)}(z, y, x)$, and Figure 1(h), $T_{\alpha, \mu \nu, \rho \sigma}^{(3)}(z, y, x)$. Again, we need a diagram involving photons in order to choose the previously introduced finite gauge. Also, these 3 diagrams clearly correspond to 3 distinct calculations.

The amplitudes of the 3 vertex correction subgraphs in these diagrams are the same as in [5]. Therefore we already know that each contribution has a logarithmic divergent Fourier transform, and that the sum of the divergent contributions from these 3 vertex subgraphs is proportional to $-2 f^{2}-g^{2}(1-\Gamma)$, therefore vanishing in the same gauge that makes the self-energy finite. Henceforth we shall use this gauge.

Let us then proceed with the Ward identity calculation, by summarizing the result from [5]. From the amplitudes for the vertex subgraphs in the diagrams $T_{\alpha, \mu \nu, \rho \sigma}^{(i)}(z, y, x), i=1,2,3$, we obtain the Ward identity for the theory [5],

$$
\frac{\partial}{\partial z_{\alpha}} T_{\alpha}(z, u, v)=-i \frac{1}{2} g \gamma_{5}\left(\delta^{4}(z-u)-\delta^{4}(z-v)\right) \Sigma(u-v)
$$

where $T_{\alpha}=\sum_{i=1}^{3} T_{\alpha}^{i}$, and $T_{\alpha}^{i}$ is the vertex subgraph in the diagram $T_{\alpha, \mu \nu, \rho \sigma}^{(i)}$. The constant $a$ in the self-energy (3.13) can be calculated as in [5] - where basically one works out the LHS in (3.14) (in the finite gauge) in order to find the correct value for (3.13) in the RHS -, and the final answer is given by

$$
\Sigma(z)=\frac{3}{64 \pi^{2}}\left(f^{2}-\frac{1}{2} g^{2}\right) \not \partial \delta^{4}(z) .
$$

Strictly speaking, one should now proceed to verify that the exact same result is obtained from the Ward identity associated with the vertex correction diagrams at the energy-momen- 
tum tensor insertions, Figures 1(i), 1(j) and 1(k). This is in fact true, but for pedagogical reasons we shall postpone such a proof for a couple of pages.

It is this result for $\Sigma(v-u)$ which is to be used to evaluate the local self-energy renormalization, Figure 1(o), therefore yielding the correct value for $a$ in (3.13). These again are 3 diagrams that amount to 1 independent calculation as in Figures 1(d) and 1(e). As (3.15) is purely local, the integral in $u$ and $v$ required for the previous diagram is trivial, simply yielding a multiple of the one loop triangle amplitude. The final result is that the sum of the self-energy insertion diagrams, Figures 1(d), 1(e) and 1(o), is a multiple of the one loop amplitude,

$$
\Sigma_{\alpha, \mu \nu, \rho \sigma}^{\prime}(z, y, x)=\frac{3}{64 \pi^{2}}\left(f^{2}-\frac{1}{2} g^{2}\right) B_{\alpha, \mu \nu, \rho \sigma}(z, y, x)
$$

exactly like in [0] as the internal fields are the same. Now recall that there is a factor of 3 from the triangular symmetry. There is also a factor of 2 for opposite directions of fermion charge flow (such term was absent in the non-planar diagrams). Finally, we are interested in the $\mathcal{O}\left(g f^{2} k^{2}\right)$ corrections, so that the term in $g^{2}$ in (3.16) should be discarded. The overall result for the self-energy contribution to the two loop correlator is finally,

$$
\Sigma_{\alpha, \mu \nu, \rho \sigma}(0, y, x)=\frac{9 f^{2}}{32 \pi^{2}} B_{\alpha, \mu \nu, \rho \sigma}(0, y, x)
$$

where we have set $z=0$ (for coherence with the other diagram calculations).

\section{Diagrams in Figures 1(f), 1(g) and 1(h)}

We can now proceed the calculation of the vertex correction diagrams at the axial current insertion, $T_{\alpha, \mu \nu, \rho \sigma}^{(i)}(z, y, x), i=1,2,3$. As for the self-energy, the calculation of these 3 diagrams follows from [5]. We shall regard each virtual photon diagram as the sum of two graphs, one with the photon propagator in the Landau gauge $\Gamma=1$, and the second with inversion covariant pure gauge propagator,

$$
\tilde{\Delta}_{\mu \nu}(u-v)=-\frac{1}{4 \pi^{2}} \frac{f^{2}}{g^{2}} \frac{1}{(u-v)^{2}} J_{\mu \nu}(u-v) .
$$


The Landau gauge diagrams give order $\mathcal{O}\left(g^{3} k^{2}\right)$ contributions to the two loop correlator, while the remainder gives an order $\mathcal{O}\left(g f^{2} k^{2}\right)$ contribution which is what we are interested in. Therefore - and similarly to what was done from (3.16) to (3.17) - we shall discard the Landau gauge diagrams from our final result, and only use (3.18) for the virtual photon propagator in the finite gauge.

With this in mind we turn to the calculation of the diagrams $T_{\alpha, \mu \nu, \rho \sigma}^{(i)}(z, y, x)$. The method of calculation is similar to the one used for the non-planar diagrams, and so we shall follow it here without giving details. After summing over both directions of Higgs field propagation, and setting $z=0$, the amplitude for $T_{\alpha, \mu \nu, \rho \sigma}^{(1)}(0, y, x)$ is written as,

$$
\begin{gathered}
T_{\alpha, \mu \nu, \rho \sigma}^{(1)}(0, y, x)=-\frac{i g f^{2} k^{2}}{8 \pi^{4}} \int d^{4} u d^{4} v \Delta(v-u) \cdot \\
\cdot \operatorname{Tr} \frac{\psi}{u^{4}} \gamma_{\alpha} \gamma_{5} \frac{\psi}{v^{4}} S(v-y) \gamma_{(\mu} \delta_{\nu) \beta}\left(\frac{\vec{\partial}}{\partial y_{\beta}}-\frac{\overleftarrow{\partial}}{\partial y_{\beta}}\right) S(y-x) \gamma_{(\rho} \delta_{\sigma) \pi}\left(\frac{\vec{\partial}}{\partial x_{\pi}}-\frac{\overleftarrow{\partial}}{\partial x_{\pi}}\right) S(x-u),
\end{gathered}
$$

and performing the conformal inversion we are led to the result,

$$
\begin{gathered}
T_{\alpha, \mu \nu, \rho \sigma}^{(1)}(0, y, x)=-\frac{i g f^{2} k^{2}}{8 \pi^{4}} y^{\prime 8} x^{\prime 8} J_{\bar{\mu}(\mu}\left(y^{\prime}\right) J_{\nu) \bar{\nu}}\left(y^{\prime}\right) J_{\bar{\rho}(\rho}\left(x^{\prime}\right) J_{\sigma) \bar{\sigma}}\left(x^{\prime}\right) \int d^{4} u^{\prime} d^{4} v^{\prime} \Delta\left(v^{\prime}-u^{\prime}\right) \\
\cdot \operatorname{Tr} \gamma_{5} \gamma_{\alpha} S\left(v^{\prime}-y^{\prime}\right) \gamma_{\bar{\mu}}\left(\frac{\vec{\partial}}{\partial y_{\bar{\nu}}^{\prime}}-\frac{\overleftarrow{\partial}}{\partial y_{\bar{\nu}}^{\prime}}\right) S\left(y^{\prime}-x^{\prime}\right) \gamma_{\bar{\rho}}\left(\frac{\vec{\partial}}{\partial x_{\bar{\sigma}}^{\prime}}-\frac{\overleftarrow{\partial}}{\partial x_{\bar{\sigma}}^{\prime}}\right) S\left(x^{\prime}-u^{\prime}\right) .
\end{gathered}
$$

As usual the expected tensorial structure emerges. We are left with the integrations to be performed. However, as we have seen, this result is divergent; only when we sum the 3 diagrams $T_{\alpha, \mu \nu, \rho \sigma}^{(i)}(z, y, x), i=1,2,3$ the result will be finite, in the finite gauge. So at this stage we should include in the calculation (3.20) the equivalent results coming from the diagrams $T_{\alpha, \mu \nu, \rho \sigma}^{(2)}(0, y, x)$ and $T_{\alpha, \mu \nu, \rho \sigma}^{(3)}(0, y, x)$ - where for this last one we should use only the inversion covariant pure gauge propagator (3.18). The result of including the 3 diagrams all together is to produce an integral of a traceless tensor, which is convergent, and can be read from the formulas in the Appendix. Hence we can write for the net sum of vertex insertions at point $z$, i.e., $T_{\alpha, \mu \nu, \rho \sigma}^{(1)}(0, y, x)$ plus $T_{\alpha, \mu \nu, \rho \sigma}^{(2)}(0, y, x)$ plus $T_{\alpha, \mu \nu, \rho \sigma}^{(3)}(0, y, x)$,

$$
T_{\alpha, \mu \nu, \rho \sigma}^{\prime}(0, y, x)=\frac{i g f^{2} k^{2}}{256 \pi^{8}} y^{\prime 8} x^{\prime 8} J_{\bar{\mu}(\mu}\left(y^{\prime}\right) J_{\nu) \bar{\nu}}\left(y^{\prime}\right) J_{\bar{\rho}(\rho}\left(x^{\prime}\right) J_{\sigma) \bar{\sigma}}\left(x^{\prime}\right) \varepsilon_{\alpha \kappa \bar{\mu} \bar{\rho}} \frac{\partial^{2}}{\partial y_{\bar{\nu}}^{\prime} \partial x_{\bar{\sigma}}^{\prime}} \frac{\left(x^{\prime}-y^{\prime}\right)_{\kappa}}{\left(x^{\prime}-y^{\prime}\right)^{4}},
$$


which is a multiple of the triangle one loop amplitude (2.18). Recalling that there is a factor of 2 for opposite directions of fermion charge flow, we can finally write for the contribution of the vertex correction diagrams (at the axial current insertion) to the two loop correlator,

$$
T_{\alpha, \mu \nu, \rho \sigma}(0, y, x)=\frac{f^{2}}{32 \pi^{2}} B_{\alpha, \mu \nu, \rho \sigma}(0, y, x)
$$

\section{Diagrams in Figures 2(a) and 2(b)}

In order to obtain the previous result we had to use finite gauge virtual photons, as given by the propagator (3.18). If one has a diagram with an internal virtual photon, one should expect a factor of $g$ from each of the two internal vertices, and so an overall contribution of order $\mathcal{O}\left(g^{3} k^{2}\right)$. However, if one uses an internal finite gauge virtual photon, there is an extra factor of $f^{2} / g^{2}$ from the propagator (3.18), and we therefore obtain an overall contribution of order $\mathcal{O}\left(g f^{2} k^{2}\right)$, which is the order we are interested in. This means that one now has to include all diagrams with one internal finite gauge photon (3.18).

In particular we have to include one more vertex in our rules, that completes (2.9-13). This vertex can be read from the action (2.1) when coupled to gravity, and is the following,

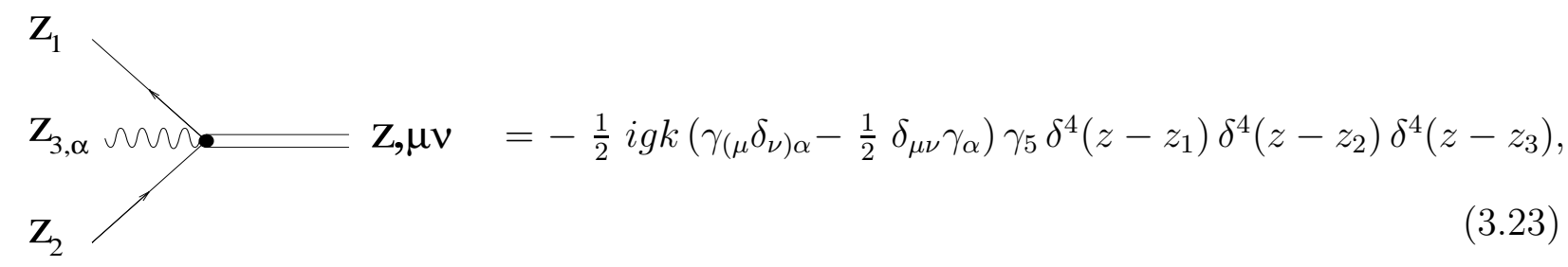

where the notation is as in (2.9-13). Observe that there is no similar vertex involving two scalar legs (as opposed to the two fermion legs we have) as such vertex would give diagrams that do not contribute to the abnormal parity part of the correlator we are computing.

When this vertex is considered, one finds that there are 7 new diagrams that must be included in our calculation, the ones presented in Figure 2. There is 1 primitive diagram in Figure 2(a). There are 2 other primitive diagrams which only correspond to 1 independent calculation, the one depicted in Figure 2(b). Then we have energy-momentum insertion 
vertex corrections. These are Figure 2(c) and Figure 2(d), 2 independent calculations corresponding to 4 diagrams due to reflection symmetry. We shall now proceed to evaluate these diagrams.

We start with the primitive diagram in Figure 2(a), $P_{\alpha, \mu \nu, \rho \sigma}^{\prime(1)}(z, y, x)$. This diagram is easily evaluated as it involves no integrations. Recall that we have to use (3.18) alone, whenever one encounters a virtual photon. Setting $z=0$ and performing the conformal inversion, the amplitude $P_{\alpha, \mu \nu, \rho \sigma}^{\prime(1)}(0, y, x)$ becomes,

$P_{\alpha, \mu \nu, \rho \sigma}^{\prime(1)}(0, y, x)=-\frac{i g f^{2} k^{2}}{64 \pi^{8}} y^{\prime 8} x^{\prime 8} J_{\bar{\mu}(\mu}\left(y^{\prime}\right) J_{\nu) \bar{\nu}}\left(y^{\prime}\right) J_{\bar{\rho}(\rho}\left(x^{\prime}\right) J_{\sigma) \bar{\sigma}}\left(x^{\prime}\right) \varepsilon_{\alpha \kappa \bar{\mu} \bar{\rho}} \frac{\left(x^{\prime}-y^{\prime}\right)_{\kappa}}{\left(x^{\prime}-y^{\prime}\right)^{6}} J_{\bar{\nu} \bar{\sigma}}\left(x^{\prime}-y^{\prime}\right)$.

Unlike all the preceeding calculations, this amplitude involves no derivatives. This is certainly to be expected due to the nature of vertex (3.23). However, one can manipulate (3.24) in order to write it as the second derivative of a tensor involving the structures (3.6) and (3.7) alone. After some calculations, one can show that (3.24) can be re-written as (including the factor of 2 for opposite directions of fermion charge flow):

$$
\begin{gathered}
P_{\alpha, \mu \nu, \rho \sigma}^{(1)}(0, y, x)=\frac{i g f^{2} k^{2}}{128 \pi^{8}} y^{\prime 8} x^{\prime 8} J_{\bar{\mu}(\mu}\left(y^{\prime}\right) J_{\nu) \bar{\nu}}\left(y^{\prime}\right) J_{\bar{\rho}(\rho}\left(x^{\prime}\right) J_{\sigma) \bar{\sigma}}\left(x^{\prime}\right) \\
\cdot\left\{\varepsilon_{\alpha \kappa \bar{\mu} \bar{\rho}} \frac{1}{\left(x^{\prime}-y^{\prime}\right)^{2}}\left(\frac{\vec{\partial}}{\partial x_{\bar{\sigma}}^{\prime}}-\frac{\overleftarrow{\partial}}{\partial x_{\bar{\sigma}}^{\prime}}\right) \frac{\left(x^{\prime}-y^{\prime}\right)_{\kappa}}{\left(x^{\prime}-y^{\prime}\right)^{2}}\left(\frac{\vec{\partial}}{\partial y_{\bar{\nu}}^{\prime}}-\frac{\overleftarrow{\partial}}{\partial y_{\bar{\nu}}^{\prime}}\right)\right\}
\end{gathered}
$$

and this tensor structure is precisely the same as the one in the non-planar diagrams (3.12). This should not come as a surprise as this diagram - like the non-planar ones - is a primitive.

Let us proceed with the primitive diagram in Figure 2(b), $P_{\alpha, \mu \nu, \rho \sigma}^{\prime(2)}(z, y, x)$. This diagram involves only one integration, therefore being different from the ones we previously calculated (which either involved two or none integrations). Upon setting $z=0$ the amplitude for $P_{\alpha, \mu \nu, \rho \sigma}^{\prime(2)}(0, y, x)$ is,

$$
\begin{gathered}
P_{\alpha, \mu \nu, \rho \sigma}^{\prime(2)}(0, y, x)=-\frac{i g f^{2} k^{2}}{128 \pi^{6}} \int \frac{d^{4} u}{(u-y)^{2}} J_{\tau \beta}(u-y) \cdot \\
\cdot \operatorname{Tr} \gamma_{\beta} \frac{\psi}{u^{4}} \gamma_{\alpha} \gamma_{5} \frac{y}{y^{4}}\left(\gamma_{(\mu} \delta_{\nu) \tau}-\frac{1}{2} \delta_{\mu \nu} \gamma_{\tau}\right) S(y-x) \gamma_{(\rho} \delta_{\sigma) \pi}\left(\frac{\vec{\partial}}{\partial x_{\pi}}-\frac{\overleftarrow{\partial}}{\partial x_{\pi}}\right) S(x-u)
\end{gathered}
$$


and performing the conformal inversion one obtains,

$$
\begin{gathered}
P_{\alpha, \mu \nu, \rho \sigma}^{\prime(2)}(0, y, x)=\frac{i g f^{2} k^{2}}{128 \pi^{6}} y^{\prime 8} x^{\prime 8} J_{\bar{\mu}(\mu}\left(y^{\prime}\right) J_{\nu) \bar{\nu}}\left(y^{\prime}\right) J_{\bar{\rho}(\rho}\left(x^{\prime}\right) J_{\sigma) \bar{\sigma}}\left(x^{\prime}\right) . \\
\cdot \int \frac{d^{4} u^{\prime}}{\left(u^{\prime}-y^{\prime}\right)^{2}} J_{\bar{\nu} \beta}\left(u^{\prime}-y^{\prime}\right) \operatorname{Tr} \gamma_{\beta} \gamma_{\alpha} \gamma_{5} \gamma_{\bar{\mu}} S\left(y^{\prime}-x^{\prime}\right) \gamma_{\bar{\rho}}\left(\frac{\vec{\partial}}{\partial x^{\prime} \bar{\sigma}}-\frac{\overleftarrow{\partial}}{\partial x_{\bar{\sigma}}^{\prime}}\right) S\left(x^{\prime}-u^{\prime}\right) .
\end{gathered}
$$

We are left with one integration to perform. However, one should note that there is only one differential operator in (3.27), and if we are to obtain a final result for this diagram which involves the tensor structures (3.6) and (3.7) we shall have to manipulate (3.27) in order to re-write it in such a way that it involves two differential operators. This is analogous to the situation we faced from (3.24) to (3.25). After integrating and performing some calculations, one obtains:

$$
\begin{array}{r}
P_{\alpha, \mu \nu, \rho \sigma}^{\prime(2)}(0, y, x)=\frac{i g f^{2} k^{2}}{128 \pi^{8}} y^{\prime 8} x^{\prime 8} J_{\bar{\mu}(\mu}\left(y^{\prime}\right) J_{\nu) \bar{\nu}}\left(y^{\prime}\right) J_{\bar{\rho}(\rho}\left(x^{\prime}\right) J_{\sigma) \bar{\sigma}}\left(x^{\prime}\right) \varepsilon_{\alpha \kappa \bar{\mu} \bar{\rho}} \\
\cdot\left\{\frac{1}{2} \frac{\partial^{2}}{\partial y_{\bar{\nu}}^{\prime} \partial x_{\bar{\sigma}}^{\prime}} \frac{\left(x^{\prime}-y^{\prime}\right)_{\kappa}}{\left(x^{\prime}-y^{\prime}\right)^{4}}+\frac{1}{\left(x^{\prime}-y^{\prime}\right)^{2}}\left(\frac{\vec{\partial}}{\partial x^{\prime}{ }_{\bar{\sigma}}}-\frac{\overleftarrow{\partial}}{\partial x_{\bar{\sigma}}^{\prime}}\right) \frac{\left(x^{\prime}-y^{\prime}\right)_{\kappa}}{\left(x^{\prime}-y^{\prime}\right)^{2}}\left(\frac{\vec{\partial}}{\partial y_{\bar{\nu}}^{\prime}}-\frac{\overleftarrow{\partial}}{\partial y_{\bar{\nu}}^{\prime}}\right)\right\}
\end{array}
$$

It is interesting to observe that this tensor structure is a linear combination of both (3.6) and (3.7). Also, one must now include in this result a factor of 2 for opposite directions of fermion charge flow and another factor of 2 associated to the 2 distinctive diagrams connected through reflection symmetry.

Finally, we can add the 3 diagrams in Figures 2(a) and 2(b), in order to obtain the primitive planar contribution to the two loop correlator,

$$
\begin{gathered}
P_{\alpha, \mu \nu, \rho \sigma}(0, y, x)=\frac{i g f^{2} k^{2}}{32 \pi^{8}} y^{\prime 8} x^{\prime 8} J_{\bar{\mu}(\mu}\left(y^{\prime}\right) J_{\nu) \bar{\nu}}\left(y^{\prime}\right) J_{\bar{\rho}(\rho}\left(x^{\prime}\right) J_{\sigma) \bar{\sigma}}\left(x^{\prime}\right) \varepsilon_{\alpha \kappa \bar{\mu} \bar{\rho}} \\
\cdot\left\{\frac{1}{2} \frac{\partial^{2}}{\partial y_{\bar{\nu}}^{\prime} \partial x_{\bar{\sigma}}^{\prime}} \frac{\left(x^{\prime}-y^{\prime}\right)_{\kappa}}{\left(x^{\prime}-y^{\prime}\right)^{4}}+\frac{5}{4} \frac{1}{\left(x^{\prime}-y^{\prime}\right)^{2}}\left(\frac{\vec{\partial}}{\partial x_{\bar{\sigma}}^{\prime}}-\frac{\overleftarrow{\partial}}{\partial x_{\bar{\sigma}}^{\prime}}\right) \frac{\left(x^{\prime}-y^{\prime}\right)_{\kappa}}{\left(x^{\prime}-y^{\prime}\right)^{2}}\left(\frac{\vec{\partial}}{\partial y_{\bar{\nu}}^{\prime}}-\frac{\overleftarrow{\partial}}{\partial y_{\bar{\nu}}^{\prime}}\right)\right\} .
\end{gathered}
$$

\section{E. Diagrams in Figures 1(i), 1(j), 1(k), 1(p), 2(c) and 2(d)}

Next, we proceed with the evaluation of the contributions coming from the vertex correction diagrams at the energy-momentum tensor insertions. These amplitudes are presented in 
Figure 1(i): $V_{\alpha, \mu \nu, \rho \sigma}^{(1)}(z, y, x)$, Figure $1(\mathrm{j}): V_{\alpha, \mu \nu, \rho \sigma}^{(2)}(z, y, x)$, Figure $1(\mathrm{k}): V_{\alpha, \mu \nu, \rho \sigma}^{(3)}(z, y, x)$, Figure 1(p): $V_{\alpha, \mu \nu, \rho \sigma}^{(4)}(z, y, x)$, and also Figure 2(c): $V_{\alpha, \mu \nu, \rho \sigma}^{(5)}(z, y, x)$, and Figure $2(\mathrm{~d}): V_{\alpha, \mu \nu, \rho \sigma}^{(6)}(z, y, x)$. Using the previous treatment with the axial insertion vertex we should insert the photon propagator in our calculation in order to guarantee finiteness of the energy-momentum insertion vertex. The amplitudes $V^{(5)}$ and $V^{(6)}$ have a different structure from the other vertex diagrams as they are semi-local. Moreover, there is a possible local term, $V^{(4)}$, which is left ambiguous by the Feynman rules. This is analogous to the situation we faced when dealing with the self-energy diagrams. As this local term cannot be evaluated by Feynman rules it will be solely determined from the Ward identity. The role it plays is one of regularizing a divergence.

The sum of these amplitudes becomes finite in the finite gauge, which also guaranteed the finiteness of the axial insertion vertex. The finite part of the vertex subgraphs is a traceless tensor with respect to all three included indices, and can be written as,

$$
V_{\mu \nu}^{(\text {finite })}(y, v, u)=\frac{3 k\left(f^{2}-\frac{1}{2} g^{2}\right)}{2 \pi^{4}} \frac{1}{(y-u)^{6}} \gamma_{\kappa}\left(\frac{(y-u)_{\mu}(y-u)_{\nu}(y-u)_{\kappa}}{(y-u)^{2}}-\frac{1}{6} \delta_{(\mu \nu}(y-u)_{\kappa)}\right) \text {. }
$$

In addition, it would be thoughtful to check the Ward identity connected to these vertices. For that we express the tensor on the RHS of (3.30) in terms of the regularized traceless structure of derivatives,

$$
\left(\frac{(y-u)_{\mu}(y-u)_{\nu}(y-u)_{\kappa}}{(y-u)^{2}}-\frac{1}{6} \delta_{(\mu \nu}(y-u)_{\kappa)}\right)=-\frac{1}{48} \frac{\partial}{\partial y_{\kappa}}\left(\frac{\partial}{\partial y_{\mu}} \frac{\partial}{\partial y_{\nu}}-\frac{1}{4} \delta_{\mu \nu} \square\right) \frac{1}{(y-u)^{2}} .
$$

With this expression it is easy to derive that the following Ward identity is satisfied for the energy-momentum insertion vertex,

$$
\frac{\partial}{\partial y^{\mu}} \int d^{4} v \sum_{i=1}^{6} V_{\mu \nu}^{(i)}(y, v, u)=-k \partial_{\nu} \Sigma(y-u)
$$

where $V_{\mu \nu}^{(i)}$ is the vertex subgraph in the diagram $V_{\alpha, \mu \nu, \rho \sigma}^{(i)}$.

This Ward identity is essential for the calculation of the amplitude of Figure 1(i). It suggests that it will give a divergent result, and only when we add together the diagrams in 
Figures 1(i), 1(j), 1(k), 1(p), 2(c) and 2(d) we shall obtain a finite answer. After summing up the two possible directions of the Higgs field and setting $z=0$ due to translation invariance of the amplitude, $V_{\alpha, \mu \nu, \rho \sigma}^{(1)}(0, y, x)$ is written as,

$$
\begin{gathered}
V_{\alpha, \mu \nu, \rho \sigma}^{(1)}(0, y, x)=-\frac{i g f^{2} k^{2}}{8 \pi^{4}} \int d^{4} u d^{4} v \Delta(u-v) \\
\cdot \operatorname{Tr} S(v-y) \gamma_{(\mu} \delta_{\nu) \beta}\left(\frac{\vec{\partial}}{\partial y_{\beta}}-\frac{\overleftarrow{\partial}}{\partial y_{\beta}}\right) S(y-u) S(u-x) \gamma_{(\rho} \delta_{\sigma) \pi}\left(\frac{\vec{\partial}}{\partial x_{\pi}}-\frac{\overleftarrow{\partial}}{\partial x_{\pi}}\right) \frac{\not}{x^{4}} \gamma_{\alpha} \gamma_{5} \frac{\psi}{v^{4}}
\end{gathered}
$$

Using the conformal properties of the theory we can perform the usual inversion in the spatial variables which results to,

$$
\begin{gathered}
V_{\alpha, \mu \nu, \rho \sigma}^{(1)}(0, y, x)=\frac{i g f^{2} k^{2}}{8 \pi^{4}} y^{\prime 8} x^{\prime 8} J_{\bar{\mu}(\mu}\left(y^{\prime}\right) J_{\nu) \bar{\nu}}\left(y^{\prime}\right) J_{\bar{\rho}(\rho}\left(x^{\prime}\right) J_{\sigma) \bar{\sigma}}\left(x^{\prime}\right) \int d^{4} u^{\prime} d^{4} v^{\prime} \Delta\left(v^{\prime}-u^{\prime}\right) \\
\cdot \operatorname{Tr} \gamma_{5} S\left(v^{\prime}-y^{\prime}\right) \gamma_{\bar{\mu}}\left(\frac{\vec{\partial}}{\partial y_{\bar{\nu}}^{\prime}}-\frac{\overleftarrow{\partial}}{\partial y_{\bar{\nu}}^{\prime}}\right) S\left(y^{\prime}-u^{\prime}\right) S\left(u^{\prime}-x^{\prime}\right) \gamma_{\bar{\rho}} \frac{\overleftarrow{\partial}}{\partial x_{\bar{\sigma}}^{\prime}} \gamma_{\alpha}
\end{gathered}
$$

In order to proceed with the $u$ and $v$ integrations we should also add the diagrams of Figures $1(\mathrm{j}), 1(\mathrm{k}), 1(\mathrm{p}), 2(\mathrm{c})$ and $2(\mathrm{~d})$ to guarantee finiteness of the contribution from the energymomentum insertion vertex. Note that all these diagrams do not contribute with a finite part for the order $\mathcal{O}\left(g f^{2} k^{2}\right)$ we are interested in, but merely make the diagram 1(i) finite. This will make the integrand have a traceless form, as given in the Appendix. After some manipulations we deduce that,

$$
V_{\alpha, \mu \nu, \rho \sigma}^{\prime \prime}(0, y, x)=\frac{i g f^{2} k^{2}}{256 \pi^{8}} y^{\prime 8} x^{\prime 8} J_{\bar{\mu}(\mu}\left(y^{\prime}\right) J_{\nu) \bar{\nu}}\left(y^{\prime}\right) J_{\bar{\rho}(\rho}\left(x^{\prime}\right) J_{\sigma) \bar{\sigma}}\left(x^{\prime}\right) \varepsilon_{\alpha \kappa \bar{\mu} \bar{\rho}} \frac{\partial^{2}}{\partial y_{\bar{\nu}}^{\prime} \partial x_{\bar{\sigma}}^{\prime}} \frac{\left(x^{\prime}-y^{\prime}\right)_{\kappa}}{\left(x^{\prime}-y^{\prime}\right)^{4}}
$$

which is proportional to the triangle structure. Taking into account the 2 fermionic directions and doubling our answer for the two distinctive diagrams connected with reflection symmetry we obtain finally:

$$
V_{\alpha, \mu \nu, \rho \sigma}^{\prime}(0, y, x)=\frac{2 f^{2}}{32 \pi^{2}} B_{\alpha, \mu \nu, \rho \sigma}(0, y, x)
$$

which is similar to what we got in (3.22). So, we can add all the diagrams that represent vertex corrections (both at axial and energy-momentum insertions). The overall result of the vertex corrections contribution to the two loop correlator is,

$$
V_{\alpha, \mu \nu, \rho \sigma}(0, y, x)=\frac{3 f^{2}}{32 \pi^{2}} B_{\alpha, \mu \nu, \rho \sigma}(0, y, x) .
$$




\section{F. Diagrams in Figures 1(1), 1(m) and 1(n)}

Finally, we would like to mention the diagrams that are zero. That these diagrams vanish can be easily seen either from the fact that the fermion trace vanishes or from arguments of Lorentz symmetry. We mention these diagrams for completeness. They are the following: Figure 1(l), which are 3 diagrams that amount to 1 independent calculation, Figure $1(\mathrm{~m})$, which is only 1 diagram, and Figure 1(n), which are 2 diagrams that amount to 1 independent calculation. We have now completed the calculations for all the 36 diagrams.

\section{G. The Three Point Function}

The next and final step is to add all diagrams together, and find out what is the two loop contribution to the three point function at order $\mathcal{O}\left(g f^{2} k^{2}\right)$. Adding the results for all our di-

agrams we obtain the $\mathcal{O}\left(g f^{2} k^{2}\right)$ two loop contribution to the correlator $\left\langle A_{\alpha}(z) T_{\mu \nu}(y) T_{\rho \sigma}(x)\right\rangle$. There are 4 distinct contributions: the one from the non-planar primitive diagrams, $N_{\alpha, \mu \nu, \rho \sigma}$ $(0, y, x)$ in $(3.12)$; the one from the self-energy diagrams, $\Sigma_{\alpha, \mu \nu, \rho \sigma}(0, y, x)$ in $(3.17)$; the one from the planar primitive diagrams, $P_{\alpha, \mu \nu, \rho \sigma}(0, y, x)$ in $(3.29)$; and the one from the vertex correction diagrams, $V_{\alpha, \mu \nu, \rho \sigma}(0, y, x)$ in (3.37). Adding these 4 structures we finally obtain our result: the three point function does not vanish and consists of two independent conformal tensor structures,

$$
\begin{gathered}
N_{\alpha, \mu \nu, \rho \sigma}(0, y, x)+\Sigma_{\alpha, \mu \nu, \rho \sigma}(0, y, x)+P_{\alpha, \mu \nu, \rho \sigma}(0, y, x)+V_{\alpha, \mu \nu, \rho \sigma}(0, y, x)= \\
=\frac{i g f^{2} k^{2}}{64 \pi^{8}} y^{\prime 8} x^{\prime 8} J_{\bar{\mu}(\mu}\left(y^{\prime}\right) J_{\nu) \bar{\nu}}\left(y^{\prime}\right) J_{\bar{\rho}(\rho}\left(x^{\prime}\right) J_{\sigma) \bar{\sigma}}\left(x^{\prime}\right) \varepsilon_{\alpha \kappa \bar{\mu} \bar{\rho}} \cdot \\
\cdot\left\{7 \frac{\partial^{2}}{\partial y_{\bar{\nu}}^{\prime} \partial x_{\bar{\sigma}}^{\prime}} \frac{\left(x^{\prime}-y^{\prime}\right)_{\kappa}}{\left(x^{\prime}-y^{\prime}\right)^{4}}+\frac{11}{2} \frac{1}{\left(x^{\prime}-y^{\prime}\right)^{2}}\left(\frac{\vec{\partial}}{\partial x_{\bar{\sigma}}^{\prime}}-\frac{\overleftarrow{\partial}}{\partial x_{\bar{\sigma}}^{\prime}}\right) \frac{\left(x^{\prime}-y^{\prime}\right)_{\kappa}}{\left(x^{\prime}-y^{\prime}\right)^{2}}\left(\frac{\vec{\partial}}{\partial y_{\bar{\nu}}^{\prime}}-\frac{\overleftarrow{\partial}}{\partial y_{\bar{\nu}}^{\prime}}\right)\right\} .
\end{gathered}
$$

There is one consistency check that can be performed on this result. Namely, under the appropriate changes, one can ask: does it reduce to the result obtained in the axial gauge theory case [5]? In order to reduce (3.38) to the gauge theory case of [5] we first have to discard all diagrams involving the vertex (3.23). Then, in the other diagrams, one has to 
erase all the "graviton derivatives". Once this is done we are left with a unique conformal tensor, i.e., we are reducing the structure of our theory to the one in [5]. Finally, taking into consideration that the removal of the "graviton derivatives" also includes a factor of -2 in the non-planar contribution (due to the symmetry enhancement of this diagrams), one finds that the overall result vanishes just as it did in [5]. This shows that our result is consistent with the calculations performed for the gauge axial anomaly.

We can trace back the reason why this radiative correction does not vanish (as it does vanish in the gauge theory case [5]). This is (3.12) and (3.29), the contribution of the primitive diagrams, which is not a multiple of the one loop amplitude. The existence of two different conformal tensors in our theory is a result of the dimensionality of the correlator $\left\langle A_{\alpha}(z) T_{\mu \nu}(y) T_{\rho \sigma}(x)\right\rangle$.

Acknowledgments: We would like to thank Daniel Freedman and Kenneth Johnson for suggesting and guiding the above investigation, as well as for comments and reading of the manuscript. We would also like to thank Roman Jackiw for comments and reading of the manuscript, and Joshua Erlich for helpful remarks. One of us (R.S.) is partially supported by the Praxis XXI grant BD-3372/94 (Portugal). 


\section{APPENDIX A: DIFFERENTIAL REGULARIZATION}

In this Appendix we study the differential regularization of the one loop triangle diagram associated to the gravitational axial anomaly, Figure 1(a). As we have seen in Section II, the amplitude for this diagram is,

$$
\frac{1}{2} i g k^{2} \operatorname{Tr} \gamma_{\alpha} \gamma_{5} S(z-y) \gamma_{(\mu} \delta_{\nu) i}\left(\frac{\vec{\partial}}{\partial y_{i}}-\frac{\overleftarrow{\partial}}{\partial y_{i}}\right) S(y-x) \gamma_{(\rho} \delta_{\sigma) j}\left(\frac{\vec{\partial}}{\partial x_{j}}-\frac{\overleftarrow{\partial}}{\partial x_{j}}\right) S(x-z)
$$

We can re-write this diagram as a "generic" fermion triangle diagram, for which the bare amplitude takes the form:

$$
\operatorname{Tr} \gamma_{I I I} S(z-y) \gamma_{I I}\left(\frac{\vec{\partial}}{\partial y_{i}}-\frac{\overleftarrow{\partial}}{\partial y_{i}}\right) S(y-x) \gamma_{I}\left(\frac{\vec{\partial}}{\partial x_{j}}-\frac{\overleftarrow{\partial}}{\partial x_{j}}\right) S(x-z)
$$

As compared to (A.1), this is a slightly more general form of the amplitude we want to

regulate. For our present purposes we shall only need to concentrate on the regularization of the singular functions present in the amplitude, so we may as well just consider (A.2).

Performing the derivatives, (A.2) can be written as:

$$
\begin{gathered}
\operatorname{Tr}\left\{-\frac{\partial^{2}}{\partial y_{i} \partial x_{j}}\left(\gamma_{I I I} S(z-y) \gamma_{I I} S(y-x) \gamma_{I} S(x-z)\right)+\right. \\
+2 \gamma_{I I} \frac{\partial}{\partial y_{i}} S(y-x) \gamma_{I} \frac{\partial}{\partial x_{j}} S(x-z) \gamma_{I I I} S(z-y)+ \\
\left.+2 \gamma_{I I I} \frac{\partial}{\partial y_{i}} S(z-y) \gamma_{I I} \frac{\partial}{\partial x_{j}} S(y-x) \gamma_{I} S(x-z)\right\}= \\
=-\frac{1}{\left(4 \pi^{2}\right)^{3}} \operatorname{Tr}\left[\gamma_{I I I} \gamma_{a} \gamma_{I I} \gamma_{b} \gamma_{I} \gamma_{c}\right]\left\{-\frac{\partial^{2}}{\partial y_{i} \partial x_{j}}\left(\frac{\partial}{\partial z_{a}} \frac{1}{(z-y)^{2}} \frac{\partial}{\partial y_{b}} \frac{1}{(y-x)^{2}} \frac{\partial}{\partial x_{c}} \frac{1}{(x-z)^{2}}\right)\right\}- \\
-\frac{2}{\left(4 \pi^{2}\right)^{3}} \operatorname{Tr}\left[\gamma_{I I} \gamma_{a} \gamma_{I} \gamma_{b} \gamma_{I I I} \gamma_{c}\right]\left\{\frac{\partial}{\partial y_{i}} \frac{\partial}{\partial y_{a}} \frac{1}{(y-x)^{2}} \frac{\partial}{\partial x_{j}} \frac{\partial}{\partial x_{b}} \frac{1}{(x-z)^{2}} \frac{\partial}{\partial z_{c}} \frac{1}{(z-y)^{2}}\right\}- \\
-\frac{2}{\left(4 \pi^{2}\right)^{3}} \operatorname{Tr}\left[\gamma_{I I I} \gamma_{a} \gamma_{I I} \gamma_{b} \gamma_{I} \gamma_{c}\right]\left\{\frac{\partial}{\partial y_{i}} \frac{\partial}{\partial z_{a}} \frac{1}{(z-y)^{2}} \frac{\partial}{\partial x_{j}} \frac{\partial}{\partial y_{b}} \frac{1}{(y-x)^{2}} \frac{\partial}{\partial x_{c}} \frac{1}{(x-z)^{2}}\right\} . \quad(\mathrm{A} .3)
\end{gathered}
$$

With $x^{\prime}=x-z, y^{\prime}=y-z, \bar{a}=c, \bar{b}=a$ and $\bar{c}=b$, we can re-write the first term in the previous result as the singular function,

$$
t_{i j \bar{a} \bar{b} \bar{c}}^{(1)}\left(x^{\prime}, y^{\prime}\right)=\frac{\partial^{2}}{\partial y_{i}^{\prime} \partial x_{j}^{\prime}}\left(\frac{\partial}{\partial x_{\bar{a}}^{\prime}} \frac{1}{x^{\prime 2}} \frac{\partial}{\partial y_{\bar{b}}^{\prime}} \frac{1}{y^{\prime 2}} \frac{\partial}{\partial x_{\bar{c}}^{\prime}} \frac{1}{\left(x^{\prime}-y^{\prime}\right)^{2}}\right),
$$

though in the following we shall drop primes and bars, and simply use the notation $(x, y)$ and $\{a, b, c\}$. By a similar re-naming of variables one can likewise manipulate the second 
and third terms in (A.3) to obtain - in both cases - the singular function,

$$
t_{i j}^{(2)}{ }_{a b c}(x, y)=\frac{\partial}{\partial x_{j}} \frac{\partial}{\partial x_{a}} \frac{1}{x^{2}} \frac{\partial}{\partial y_{i}} \frac{\partial}{\partial y_{b}} \frac{1}{y^{2}} \frac{\partial}{\partial x_{c}} \frac{1}{(x-y)^{2}}
$$

Observe that (A.5) is independent of (A.4), so that in (A.3) we have two independent singular functions. For particular choices of the vertex gamma matrices, (A.2) describes the anomalous three point function (explicitly studied in the paper) as well as other physically interesting amplitudes.

We need to "pull out" derivatives and regularize both $t_{i j}^{(1)}{ }_{a b c}(x, y)$ and $t_{i j}^{(2)}{ }_{a b c}(x, y)$. Starting with (A.4), one easily observes that this can be written as,

$$
t_{i j}^{(1)}{ }_{a b c}(x, y)=\frac{\partial^{2}}{\partial y_{i} \partial x_{j}} t_{a b c}(x, y),
$$

where $t_{a b c}(x, y)$ is the singular function which is present in the bare amplitude for the gauge axial anomaly, and has been previously considered in [10]. In particular, it was shown in this reference how to regularize this singular function. Two derivatives are required to control the linear divergence arising from the singularity at $x \sim y \sim 0$. Making manifest the $x \leftrightarrow y$, $a \leftrightarrow b$ antisymmetry of $t_{a b c}(x, y)$ one can write,

$$
t_{a b c}(x, y)=F_{a b c}(x, y)+S_{a b c}(x, y)
$$

where the function $F_{a b c}(x, y)$ has finite Fourier transform by power counting and trace arguments,

$$
\begin{gathered}
F_{a b c}(x, y)=\frac{\partial}{\partial x_{a}} \frac{\partial}{\partial y_{b}}\left[\frac{1}{x^{2} y^{2}} \frac{\partial}{\partial x_{c}} \frac{1}{(x-y)^{2}}\right]+\frac{\partial}{\partial x_{a}}\left[\frac{1}{x^{2} y^{2}}\left(\frac{\partial^{2}}{\partial x_{b} \partial x_{c}}-\frac{1}{4} \delta_{b c} \square\right) \frac{1}{(x-y)^{2}}\right]- \\
-\frac{\partial}{\partial y_{b}}\left[\frac{1}{x^{2} y^{2}}\left(\frac{\partial^{2}}{\partial x_{a} \partial x_{c}}-\frac{1}{4} \delta_{a c} \square\right) \frac{1}{(x-y)^{2}}\right]- \\
-\frac{1}{x^{2} y^{2}}\left[\frac{\partial^{3}}{\partial x_{a} \partial x_{b} \partial x_{c}}-\frac{1}{6}\left(\delta_{a b} \frac{\partial}{\partial x_{c}}+\delta_{b c} \frac{\partial}{\partial x_{a}}+\delta_{a c} \frac{\partial}{\partial x_{b}}\right) \square\right] \frac{1}{(x-y)^{2}} .
\end{gathered}
$$

The term $S_{a b c}(x, y)$ contains the "traces" subtracted off in (A.8) and thus derivatives of $\delta(x-y)$ times $1 / x^{4}$ factors which are regulated as is standard in differential regularization, 
yielding:

$$
\begin{gathered}
S_{a b c}(x, y)=\frac{1}{4} \pi^{2}\left\{\left[\delta_{b c} \frac{\partial}{\partial x_{a}}-\delta_{a c} \frac{\partial}{\partial y_{b}}\right] \delta(x-y) \square \frac{\ln M_{1}^{2} x^{2}}{x^{2}}-\right. \\
\left.-\frac{1}{3}\left[\delta_{b c}\left(\frac{\partial}{\partial x_{a}}-\frac{\partial}{\partial y_{a}}\right)+\delta_{a c}\left(\frac{\partial}{\partial x_{b}}-\frac{\partial}{\partial y_{b}}\right)+\delta_{a b}\left(\frac{\partial}{\partial x_{c}}-\frac{\partial}{\partial y_{c}}\right)\right] \delta(x-y) \square \frac{\ln M_{2}^{2} x^{2}}{x^{2}}\right\} .
\end{gathered}
$$

Two different mass scales were used for the two independent trace terms in $S_{a b c}(x, y)$. Renormalization or symmetry conditions may be used to determine the ratio $M_{1} / M_{2}$ in particular cases of the triangle amplitude.

Expressions (A.8) and (A.9) provide the required regularization of (A.4) via expressions (A.6-7). We are thus left with the regularization of (A.5), which can be performed in a similar fashion. Again, one can write,

$$
t_{i j a b c}^{(2)}(x, y)=F_{i j_{a b c}}^{\prime}(x, y)+S_{i j_{a b c}}^{\prime}(x, y) .
$$

The function $F_{i j_{a b c}}^{\prime}(x, y)$ whose Fourier transform is finite by power counting and trace arguments is,

$$
\begin{gathered}
F_{i j_{a b c}}^{\prime}(x, y)=\frac{\partial}{\partial y_{i}} \frac{\partial}{\partial x_{j}} \frac{\partial}{\partial x_{a}} \frac{\partial}{\partial y_{b}}\left[\frac{1}{x^{2} y^{2}} \frac{\partial}{\partial x_{c}} \frac{1}{(x-y)^{2}}\right]+\frac{\partial}{\partial y_{i}} \frac{\partial}{\partial x_{j}} \\
\cdot\left\{\frac{\partial}{\partial x_{a}}\left[\frac{1}{x^{2} y^{2}}\left(\frac{\partial^{2}}{\partial x_{b} \partial x_{c}}-\frac{1}{4} \delta_{b c} \square\right) \frac{1}{(x-y)^{2}}\right]-\frac{\partial}{\partial y_{b}}\left[\frac{1}{x^{2} y^{2}}\left(\frac{\partial^{2}}{\partial x_{a} \partial x_{c}}-\frac{1}{4} \delta_{a c} \square\right) \frac{1}{(x-y)^{2}}\right]-\right. \\
\left.-\frac{1}{x^{2} y^{2}}\left[\frac{\partial^{3}}{\partial x_{a} \partial x_{b} \partial x_{c}}-\frac{1}{6}\left(\delta_{a b} \frac{\partial}{\partial x_{c}}+\delta_{b c} \frac{\partial}{\partial x_{a}}+\delta_{a c} \frac{\partial}{\partial x_{b}}\right) \square\right] \frac{1}{(x-y)^{2}}\right\}+ \\
+\frac{\partial}{\partial y_{i}} \frac{\partial}{\partial x_{a}}\left\{\frac{\partial}{\partial y_{b}}\left[\frac{1}{x^{2} y^{2}}\left(\frac{\partial^{2}}{\partial x_{j} \partial x_{c}}-\frac{1}{4} \delta_{j c} \square\right) \frac{1}{(x-y)^{2}}\right]-\right. \\
\left.-\frac{1}{x^{2} y^{2}}\left[\frac{\partial^{3}}{\partial x_{b} \partial x_{j} \partial x_{c}}-\frac{1}{6}\left(\delta_{b j} \frac{\partial}{\partial x_{c}}+\delta_{j c} \frac{\partial}{\partial x_{b}}+\delta_{b c} \frac{\partial}{\partial x_{j}}\right) \square\right] \frac{1}{(x-y)^{2}}\right\}- \\
-\frac{\partial}{\partial y_{b}} \frac{\partial}{\partial x_{j}}\left\{\frac{\partial}{\partial x_{a}}\left[\frac{1}{x^{2} y^{2}}\left(\frac{\partial^{2}}{\partial x_{i} \partial x_{c}}-\frac{1}{4} \delta_{i c} \square\right) \frac{1}{(x-y)^{2}}\right]-\right. \\
\left.-\frac{1}{x^{2} y^{2}}\left[\frac{\partial^{3}}{\partial x_{a} \partial x_{i} \partial x_{c}}-\frac{1}{6}\left(\delta_{a i} \frac{\partial}{\partial x_{c}}+\delta_{i c} \frac{\partial}{\partial x_{a}}+\delta_{a c} \frac{\partial}{\partial x_{i}}\right) \square\right] \frac{1}{(x-y)^{2}}\right\}- \\
-\frac{\partial}{\partial y_{i}} \frac{\partial}{\partial y_{b}}\left\{\frac{1}{x^{2} y^{2}}\left[\frac{\partial^{3}}{\partial x_{a} \partial x_{j} \partial x_{c}}-\frac{1}{6}\left(\delta_{a j} \frac{\partial}{\partial x_{c}}+\delta_{j c} \frac{\partial}{\partial x_{a}}+\delta_{a c} \frac{\partial}{\partial x_{j}}\right) \square\right] \frac{1}{(x-y)^{2}}\right\}+ \\
+\frac{\partial}{\partial x_{j}} \frac{\partial}{\partial x_{a}}\left\{\frac{1}{x^{2} y^{2}}\left[\frac{\partial^{3}}{\partial x_{i} \partial x_{b} \partial x_{c}}-\frac{1}{6}\left(\delta_{i b} \frac{\partial}{\partial x_{c}}+\delta_{b c} \frac{\partial}{\partial x_{i}}+\delta_{i c} \frac{\partial}{\partial x_{b}}\right) \square\right] \frac{1}{(x-y)^{2}}\right\}-
\end{gathered}
$$




$$
\begin{aligned}
& -\frac{\partial}{\partial x_{a}} \frac{\partial}{\partial y_{b}}\left\{\frac{1}{x^{2} y^{2}}\left[\frac{\partial^{3}}{\partial x_{i} \partial x_{j} \partial x_{c}}-\frac{1}{6}\left(\delta_{i j} \frac{\partial}{\partial x_{c}}+\delta_{j c} \frac{\partial}{\partial x_{i}}+\delta_{i c} \frac{\partial}{\partial x_{j}}\right) \square\right] \frac{1}{(x-y)^{2}}\right\}+ \\
& + \text { Terms invovling } 4^{\text {th }} \text { and } 5^{\text {th }} \text { order traceless derivatives acting on } \frac{1}{(x-y)^{2}} .
\end{aligned}
$$

The fourth and fifth derivatives can be obtained in a straightforward fashion, but their explicit form is not relevant and they would occupy a couple of pages to write down. Therefore we omit these terms.

Again, the term $S_{i j_{a b c}}^{\prime}(x, y)$ contains "traces" subtracted from (A.11) and so its structure is similar to the one of (A.9), containing the usual differential regulated derivatives of $\delta(x-y)$ times $1 / x^{4}$ factors. One obtains,

$$
\begin{gathered}
S_{i j_{a b c}}^{\prime}(x, y)=\frac{\partial}{\partial y_{i}} \frac{\partial}{\partial x_{j}} \frac{1}{4} \pi^{2}\left\{\left[\delta_{b c} \frac{\partial}{\partial x_{a}}-\delta_{a c} \frac{\partial}{\partial y_{b}}\right] \delta(x-y) \square \frac{\ln M_{1}^{2} x^{2}}{x^{2}}-\right. \\
\left.-\frac{1}{3}\left[\delta_{b c}\left(\frac{\partial}{\partial x_{a}}-\frac{\partial}{\partial y_{a}}\right)+\delta_{a c}\left(\frac{\partial}{\partial x_{b}}-\frac{\partial}{\partial y_{b}}\right)+\delta_{a b}\left(\frac{\partial}{\partial x_{c}}-\frac{\partial}{\partial y_{c}}\right)\right] \delta(x-y) \square \frac{\ln M_{2}^{2} x^{2}}{x^{2}}\right\}- \\
-\frac{\partial}{\partial y_{i}} \frac{\partial}{\partial x_{a}} \frac{1}{4} \pi^{2}\left\{\delta_{j c} \frac{\partial}{\partial y_{b}} \delta(x-y) \square \frac{\ln M_{3}^{2} x^{2}}{x^{2}}+\frac{1}{3}\left[\delta_{b c}\left(\frac{\partial}{\partial x_{j}}-\frac{\partial}{\partial y_{j}}\right)+\delta_{j c}\left(\frac{\partial}{\partial x_{b}}-\frac{\partial}{\partial y_{b}}\right)+\right.\right. \\
\left.\left.+\delta_{j b}\left(\frac{\partial}{\partial x_{c}}-\frac{\partial}{\partial y_{c}}\right)\right] \delta(x-y) \square \frac{\ln M_{4}^{2} x^{2}}{x^{2}}\right\}-\frac{\partial}{\partial y_{b}} \frac{\partial}{\partial x_{j}} \frac{1}{4} \pi^{2}\left\{\delta_{i c} \frac{\partial}{\partial x_{a}} \delta(x-y) \square \frac{\ln M_{3}^{2} x^{2}}{x^{2}}-\right. \\
\left.-\frac{1}{3}\left[\delta_{i c}\left(\frac{\partial}{\partial x_{a}}-\frac{\partial}{\partial y_{a}}\right)+\delta_{a c}\left(\frac{\partial}{\partial x_{i}}-\frac{\partial}{\partial y_{i}}\right)+\delta_{a i}\left(\frac{\partial}{\partial x_{c}}-\frac{\partial}{\partial y_{c}}\right)\right] \delta(x-y) \square \frac{\ln M_{4}^{2} x^{2}}{x^{2}}\right\}- \\
-\frac{\partial}{\partial y_{i}} \frac{\partial}{\partial y_{b}} \frac{\pi^{2}}{12}\left\{\left[\delta_{j c}\left(\frac{\partial}{\partial x_{a}}-\frac{\partial}{\partial y_{a}}\right)+\delta_{a c}\left(\frac{\partial}{\partial x_{j}}-\frac{\partial}{\partial y_{j}}\right)+\delta_{a j}\left(\frac{\partial}{\partial x_{c}}-\frac{\partial}{\partial y_{c}}\right)\right] \delta(x-y) \square \frac{\ln M_{5}^{2} x^{2}}{x^{2}}\right\}+ \\
+\frac{\partial}{\partial x_{j}} \frac{\partial}{\partial x_{a}} \frac{\pi^{2}}{12}\left\{\left[\delta_{b c}\left(\frac{\partial}{\partial x_{i}}-\frac{\partial}{\partial y_{i}}\right)+\delta_{i c}\left(\frac{\partial}{\partial x_{b}}-\frac{\partial}{\partial y_{b}}\right)+\delta_{i b}\left(\frac{\partial}{\partial x_{c}}-\frac{\partial}{\partial y_{c}}\right)\right] \delta(x-y) \square \frac{\ln M_{5}^{2} x^{2}}{x^{2}}\right\}- \\
-\frac{\partial}{\partial x_{a}} \frac{\partial}{\partial y_{b}} \frac{\pi^{2}}{12}\left\{\left[\delta_{j c}\left(\frac{\partial}{\partial x_{i}}-\frac{\partial}{\partial y_{i}}\right)+\delta_{i c}\left(\frac{\partial}{\partial x_{j}}-\frac{\partial}{\partial y_{j}}\right)+\delta_{i j}\left(\frac{\partial}{\partial x_{c}}-\frac{\partial}{\partial y_{c}}\right)\right] \delta(x-y) \square \frac{\ln M_{6}^{2} x^{2}}{x^{2}}\right\}+ \\
+\operatorname{Traces} \text { subtracted from }(\mathrm{A} .11) \text { in the } 4^{t h} \text { and } 5^{t h} \text { order derivatives, }
\end{gathered}
$$$$
\text { and their respective mass scales. }
$$

One can see that several different mass scales were introduced for the several independent trace terms in $S_{i j_{a b c}}^{\prime}(x, y)$. Again, as was mentioned for (A.9), renormalization or symmetry conditions may be used to determine the ratios between the mass scales in particular cases of the triangle amplitude. 


\section{APPENDIX B: CONVOLUTION INTEGRALS}

In this Appendix we list the convolution integrals that are required in order to perform the two loop calculation [15]. They are inclosed to make this work self contained for the

reader who wishes to reproduce our result. The table of convolution integrals is (defining $\Delta \equiv x-y$, and using the cutoff $\Lambda)$ :

$$
\begin{aligned}
& \int \frac{d^{4} v}{v^{2}(v-x)^{2}}=-\pi^{2} \ln \frac{x^{2}}{\Lambda^{2}} \\
& \int \frac{(v-x)_{\rho}}{v^{2}(v-x)^{4}} d^{4} v=-\pi^{2} \frac{x_{\rho}}{x^{2}} \\
& \int \frac{(v-x)_{\rho}(v-y)_{\sigma}}{(v-x)^{4}(v-y)^{4}} d^{4} v=\frac{\pi^{2}}{2 \Delta^{2}}\left(\delta_{\rho \sigma}-2 \frac{\Delta_{\rho} \Delta_{\sigma}}{\Delta^{2}}\right) \\
& \int \frac{\left(v_{\rho} v_{\sigma}-\frac{1}{4} \delta_{\rho \sigma} v^{2}\right)}{v^{4}(v-x)^{2}} d^{4} v=\frac{\pi^{2}}{2 x^{2}}\left(x_{\rho} x_{\sigma}-\frac{1}{4} \delta_{\rho \sigma} x^{2}\right), \\
& \int \frac{\left((v-x)_{\rho}(v-x)_{\sigma}-\frac{1}{4} \delta_{\rho \sigma}(v-x)^{2}\right)(v-y)_{\lambda}}{(v-x)^{4}(v-y)^{4}} d^{4} v= \\
& =-\frac{\pi^{2}}{4 \Delta^{2}}\left(\delta_{\rho \lambda} \Delta_{\sigma}+\delta_{\sigma \lambda} \Delta_{\rho}-2 \frac{\Delta_{\rho} \Delta_{\sigma} \Delta_{\lambda}}{\Delta^{2}}\right), \\
& \int \frac{\left(v_{\rho} v_{\sigma}-\frac{1}{4} \delta_{\rho \sigma} v^{2}\right)}{v^{6}(v-x)^{2}} d^{4} v=\frac{\pi^{2}}{2 x^{4}}\left(x_{\rho} x_{\sigma}-\frac{1}{4} \delta_{\rho \sigma} x^{2}\right), \\
& \int \frac{\left((v-x)_{\rho}(v-x)_{\sigma}-\frac{1}{4} \delta_{\rho \sigma}(v-x)^{2}\right)(v-y)_{\lambda}}{(v-x)^{6}(v-y)^{4}} d^{4} v= \\
& =-\frac{\pi^{2}}{4 \Delta^{4}}\left(\delta_{\rho \lambda} \Delta_{\sigma}+\delta_{\sigma \lambda} \Delta_{\rho}+\frac{1}{2} \delta_{\rho \sigma} \Delta_{\lambda}-4 \frac{\Delta_{\rho} \Delta_{\sigma} \Delta_{\lambda}}{\Delta^{2}}\right), \\
& \int \frac{v_{\alpha}(v-x)_{\rho}(v-y)_{\sigma}}{(v-x)^{4}(v-y)^{4}} d^{4} v=\frac{\pi^{2}}{4 \Delta^{2}}\left(\delta_{\alpha \rho} \Delta_{\sigma}-\delta_{\alpha \sigma} \Delta_{\rho}+(x+y)_{\alpha}\left[\delta_{\rho \sigma}-2 \frac{\Delta_{\rho} \Delta_{\sigma}}{\Delta^{2}}\right]\right) \\
& \int \frac{v_{\alpha}(v-y)_{\rho}}{(v-x)^{2}(v-y)^{4}} d^{4} v=\frac{\pi^{2}}{2 \Delta^{2}}\left((x+y)_{\alpha} \Delta_{\rho}-\frac{1}{4} \delta_{\alpha \rho} \Delta^{2}\right)-\frac{\pi^{2}}{4} \delta_{\alpha \rho} \ln \frac{\Delta^{2}}{\Lambda^{2}} .
\end{aligned}
$$




\section{REFERENCES}

[1] J.S. Bell and R. Jackiw, A PCAC Puzzle: $\pi^{0} \rightarrow \gamma \gamma$ in the Sigma Model, Nuovo Cim. A60 (1969) 47.

[2] S.L. Adler, Axial Vector Vertex in Spinor Electrodynamics, Phys. Rev. 177 (1969) 2426.

[3] S.L. Adler and W.A. Bardeen, Absence of Higher Order Corrections in the Anomalous Axial Vector Divergence Equation, Phys. Rev. 182 (1969) 1517.

[4] M. Baker and K. Johnson, Applications of Conformal Symmetry in Quantum Electrodynamics, Physica 96A (1979) 120.

[5] J. Erlich and D.Z. Freedman, Conformal Symmetry and the Chiral Anomaly, Phys. Rev. D55 (1997) 6522, hep-th/9611133.

[6] R. Delbourgo and A. Salam, The Gravitational Correction to PCAC, Phys. Lett. B40 (1972) 381.

[7] T. Eguchi and P.G.O. Freund, Quantum Gravity and World Topology, Phys. Rev. Lett. 37 (1976) 1251.

[8] R. Delbourgo, A Dimensional Derivation of the Gravitational PCAC Correction, J. Phys. A10 (1977) L237.

[9] L. Alvarez-Gaumé and E. Witten, Gravitational Anomalies, Nucl. Phys. B234 (1983) 269.

[10] D.Z. Freedman, K. Johnson and J.I. Latorre, Differential Regularization and Renormalization: a New Method of Calculation in Quantum Field Theory, Nucl. Phys. B371 (1992) 353

[11] E.J. Schreier, Conformal Symmetry and Three-Point Functions, Phys. Rev. D3 (1971) 980. 
[12] H. Osborn and A. Petkos, Implications of Conformal Invariance in Field Theories for General Dimensions, Annals Phys. 231 (1994) 311, hep-th/9307010.

[13] J. Erdmenger and H. Osborn, Conserved Currents and the Energy Momentum Tensor in Conformally Invariant Theories for General Dimensions, Nucl. Phys. B483 (1997) 431, hep-th/9605009.

[14] H. Sonoda, Understanding Chiral Anomaly in Coordinate Space, Phys. Rev. D55 (1997) 5245, hep-th/9608050.

[15] J.I. Rosner, Higher-Order Contributions to the Divergent Part of $Z_{3}$ in a Model Quantum Electrodynamics, Annals Phys. 44 (1967) 11. 
$\mathrm{A}_{\alpha}(\mathrm{z})$

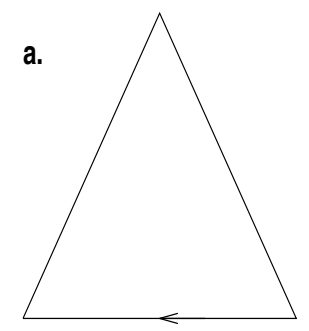

$\mathrm{T}_{\mu \nu}(\mathrm{y})$

$\mathrm{T}_{\rho \sigma}(\mathrm{x})$

$\mathrm{A}_{\alpha}(\mathrm{z})$

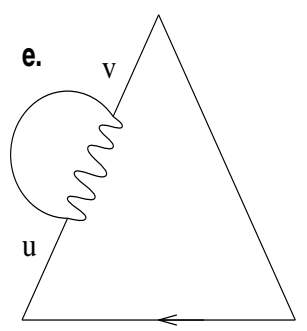

$\mathrm{T}_{\mu \nu}(\mathrm{y})$

$\mathrm{T}_{\rho \sigma}(\mathrm{x})$

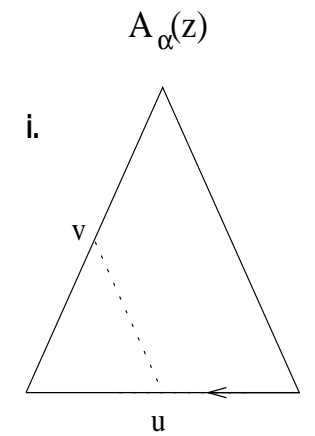

$\mathrm{T}_{\mu \nu}(\mathrm{y})$

$\mathrm{T}_{\rho \sigma}(\mathrm{x})$

$\mathrm{A}_{\alpha}(\mathrm{z})$
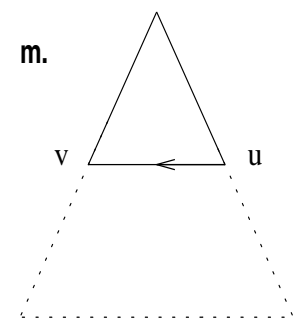

$\mathrm{T}_{\mu v}(\mathrm{y})$

$\mathrm{T}_{\rho \sigma}(\mathrm{x})$
$\mathrm{A}_{\alpha}(\mathrm{z})$

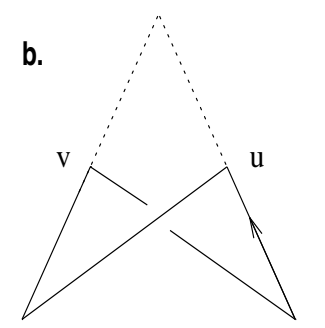

$\mathrm{T}_{\mu v}(\mathrm{y})$

$\mathrm{T}_{\rho \sigma}(\mathrm{x})$

$\mathrm{A}_{\alpha}(\mathrm{z})$

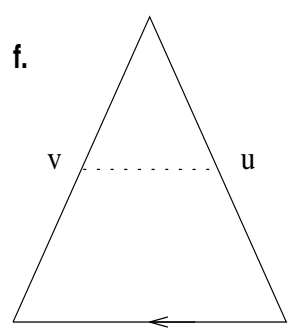

$\mathrm{T}_{\mu v}(\mathrm{y})$

$\mathrm{T}_{\rho \sigma}(\mathrm{x})$

$\mathrm{A}_{\alpha}(\mathrm{z})$

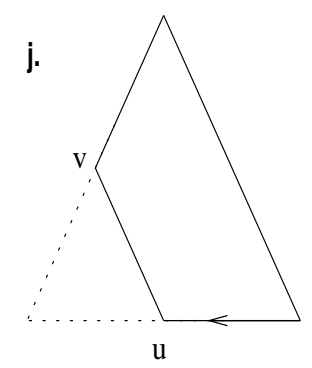

$\mathrm{T}_{\mu v}(\mathrm{y})$

$\mathrm{T}_{\rho \sigma}(\mathrm{x})$

$\mathrm{A}_{\alpha}(\mathrm{z})$

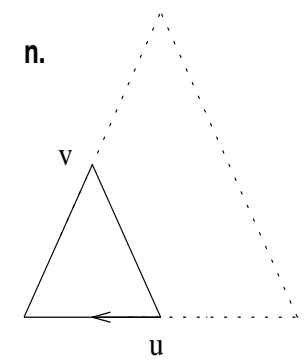

$\mathrm{T}_{\mu v}(\mathrm{y})$

$\mathrm{T}_{\rho \sigma}(\mathrm{x})$
$\mathrm{A}_{\alpha}(\mathrm{z})$

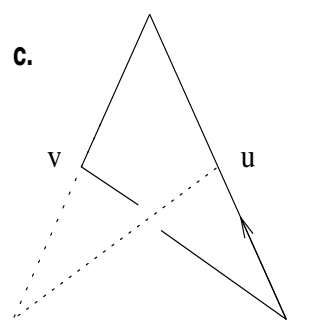

$\mathrm{T}_{\mu \nu}(\mathrm{y})$

$\mathrm{T}_{\rho \sigma}(\mathrm{x})$

$\mathrm{A}_{\alpha}(\mathrm{z})$

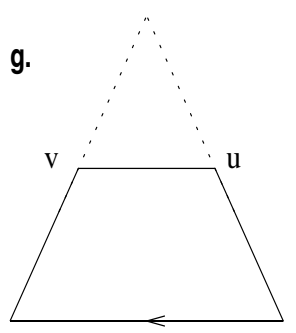

$\mathrm{T}_{\mu v}(\mathrm{y})$

$\mathrm{T}_{\rho \sigma}(\mathrm{x})$

$\mathrm{A}_{\alpha}(\mathrm{z})$

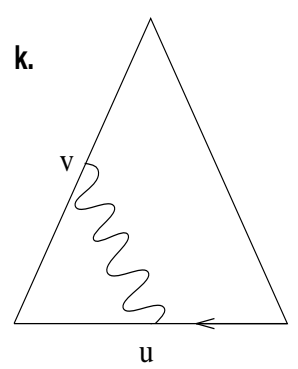

$\mathrm{T}_{\mu v}(\mathrm{y})$

$\mathrm{T}_{\rho \sigma}(\mathrm{x})$

$\mathrm{A}_{\alpha}(\mathrm{z})$

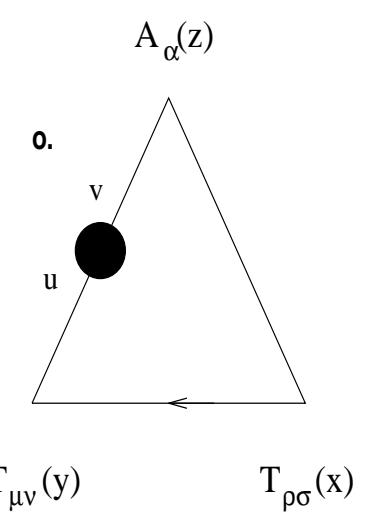

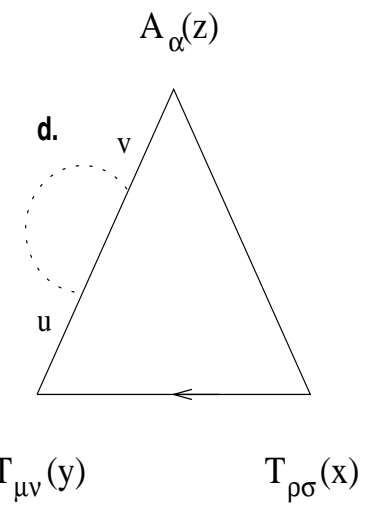
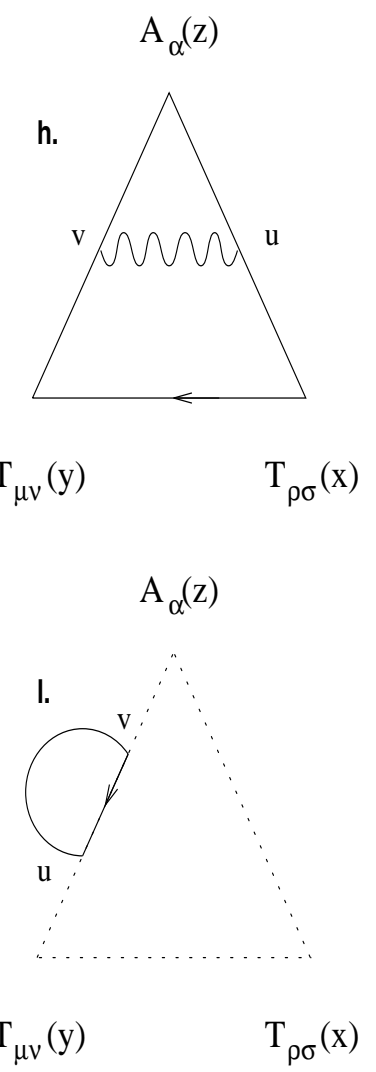

FIG. 1. One and two loop contributions to the anomaly in the Abelian Higgs theory. 

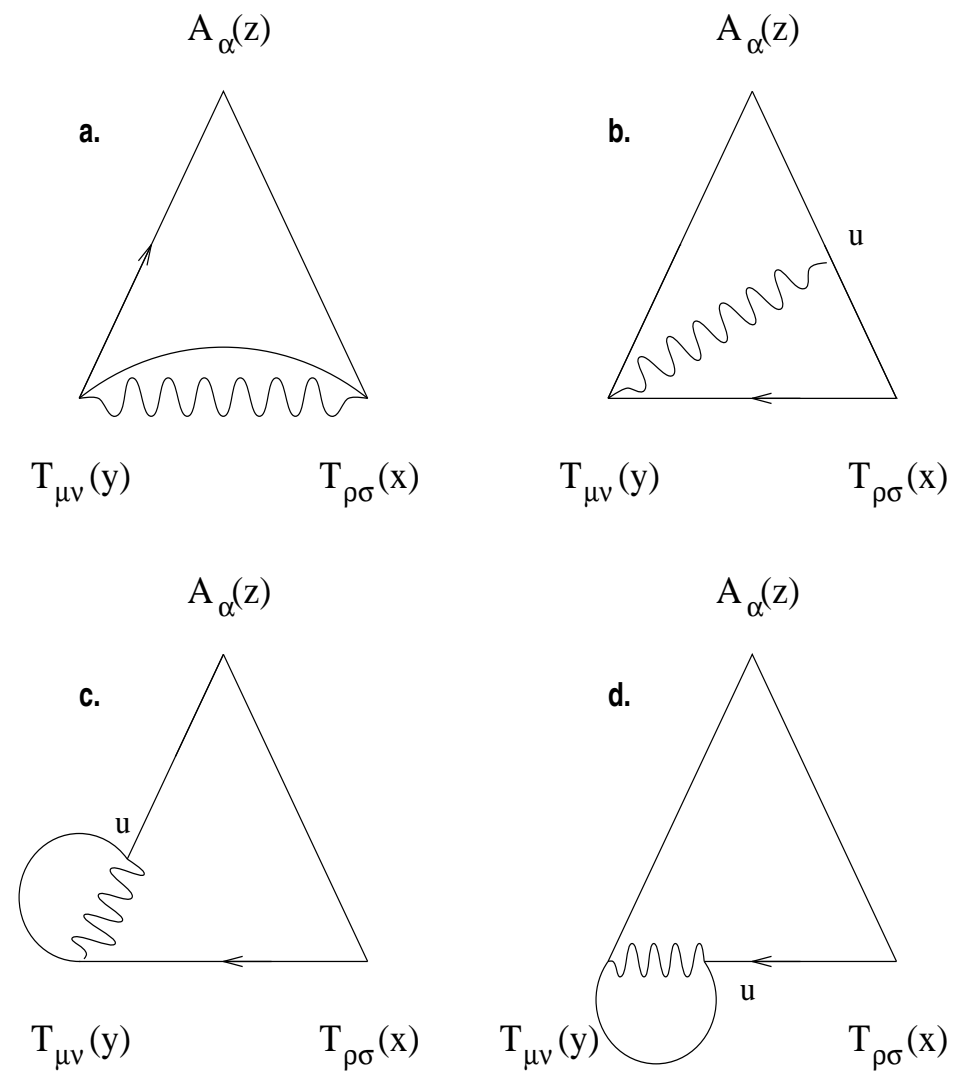

FIG. 2. Two loop contributions to the anomaly involving the four-point vertex. 